Provided for non-commercial research and education use. Not for reproduction, distribution or commercial use.

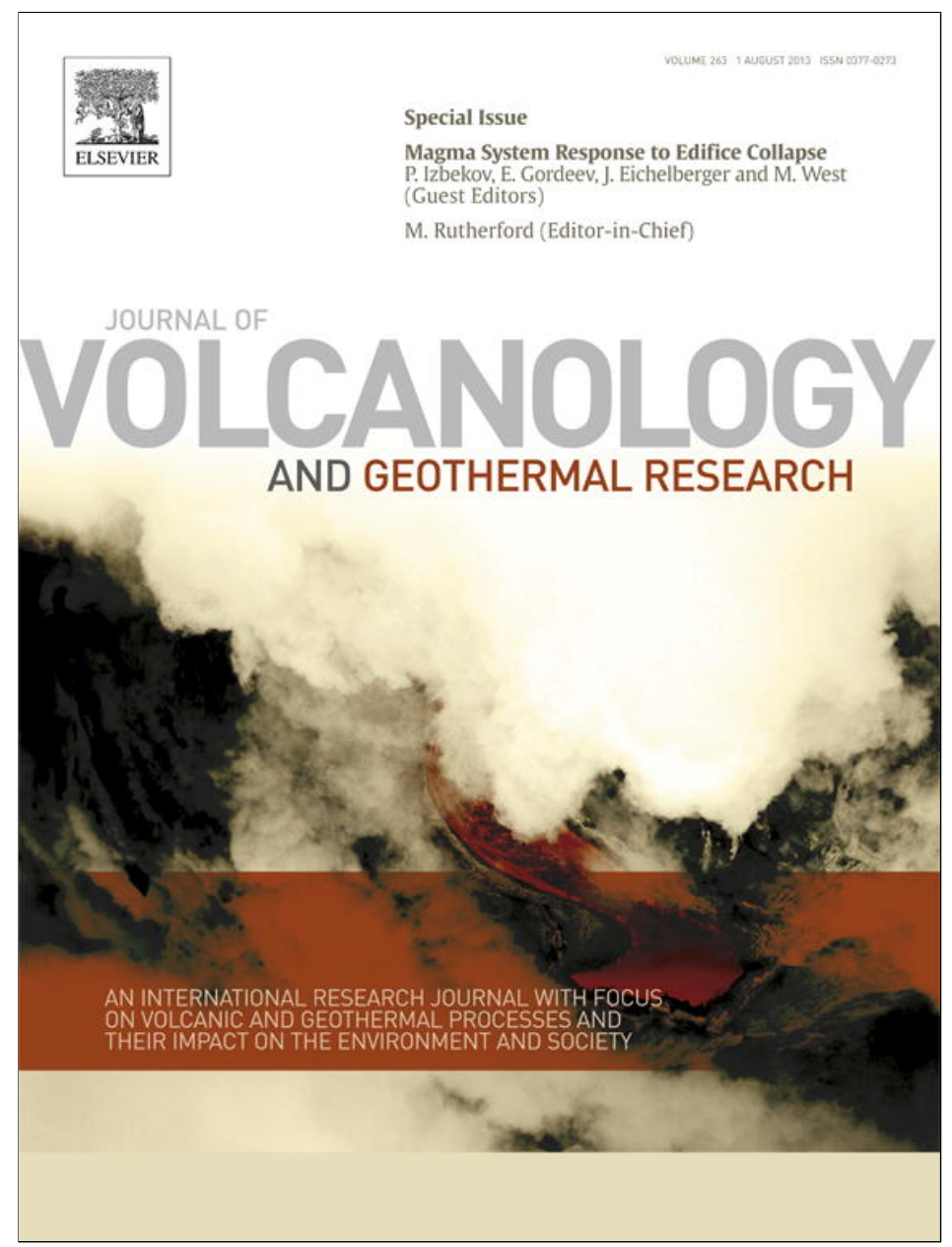

This article appeared in a journal published by Elsevier. The attached copy is furnished to the author for internal non-commercial research and education use, including for instruction at the authors institution and sharing with colleagues.

Other uses, including reproduction and distribution, or selling or licensing copies, or posting to personal, institutional or third party websites are prohibited.

In most cases authors are permitted to post their version of the article (e.g. in Word or Tex form) to their personal website or institutional repository. Authors requiring further information regarding Elsevier's archiving and manuscript policies are encouraged to visit:

http://www.elsevier.com/authorsrights 


\title{
Volcanic structure and composition of Old Shiveluch volcano, Kamchatka
}

\author{
Natalia Gorbach ${ }^{\mathrm{a}, *}$, Maxim Portnyagin ${ }^{\mathrm{b}, \mathrm{c}}$, Igor Tembrel ${ }^{\mathrm{a}}$ \\ a Institute of Volcanology and Seismology, Piip Boulevard 9, 683006, Petropavlovsk-Kamchatsky, Russia \\ b Helmholtz Centre for Ocean Research Kiel (GEOMAR), Wischhofstrasse 1-3, 24148 Kiel, Germany \\ c V.I. Vernadsky Institute of Geochemistry and Analytical Chemistry, RAS, Kosigin St. 19, 119991 Moscow, Russia
}

\section{A R T I C L E I N F O}

\section{Article history:}

Received 20 January 2012

Accepted 19 December 2012

Available online 31 December 2012

\section{Keywords:}

Kamchatka

Shiveluch

Mapping

Magnesian andesites

Crystallization

Magma mixing

Sector collapse

\begin{abstract}
A B S T R A C T
This paper reports results of a new comprehensive geological mapping of the Late Pleistocene Old Shiveluch volcano. The mapping results and geochemical data on major and trace element composition of the volcanic rocks are used to characterize spatial distribution, eruptive sequence and volumetric relationships between different rock types of the volcano. Old Shiveluch volcano had been constructed during two main stages: initial explosive and subsequent effusive ones. Pyroclastic deposits of the initial stage are represented by agglomerate and psephytic tuffs with very few lava flows and form at least $60 \%$ of volume of the Old Shiveluch edifice. The deposits of the second stage are dominantly lava flows erupted from four vents: Central, Western, Baidarny and Southern, reconstructed from the field relationships of their lava flows. About $75 \%$ of the Old Shiveluch edifice, both pyroclastic deposits and lava, are composed of magnesian andesites $\left(\mathrm{SiO}_{2}=57.3-\right.$ 63.8 wt.\%, Mg\#=0.53-0.57). The most abundant andesitic lavas were coevally erupted from the Central and Western vents in the central part of the edifice. Less voluminous high-Al basaltic andesites $\left(\mathrm{SiO}_{2}=\right.$ 53.5-55.7 wt.\%, Mg\#=0.52-0.56) were produced by the Western, Baidarny and Southern vents situated in the south-western sector. Small volume high-Mg basaltic andesites $\left(\mathrm{SiO}_{2}=53.9-55.0 \mathrm{wt} . \%, \mathrm{Mg} \#=0.59\right.$ 0.64 ) occur in the upper part of the pyroclastic deposits.

Andesites of Old and Young Shiveluch Volcanoes have similar compositions, whereas Old Shiveluch basaltic an-

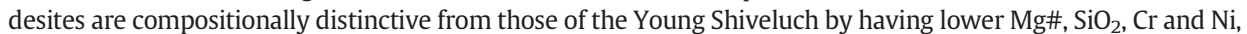
and higher $\mathrm{Al}_{2} \mathrm{O}_{3}, \mathrm{FeO}^{\mathrm{T}}, \mathrm{CaO}, \mathrm{TiO}_{2}$, and $\mathrm{V}$ contents at given MgO. Geochemical modeling suggests that the compositions of the intermediate Old Shiveluch magmas can be reasonably explained by simple fractional crystallization of olivine, clinopyroxene, plagioclase and magnetite ( \pm hornblende) from water-bearing $\left(\sim 3 \mathrm{wt} . \% \mathrm{H}_{2} \mathrm{O}\right)$ high-Mg\# basaltic parental magma at intermediate to shallow crustal depths $(<15 \mathrm{~km})$. Mixing of evolved $\left(\mathrm{SiO}_{2}>60 \mathrm{wt} . \%, \mathrm{Mg} \#<0.55\right)$ and primitive magmas $\left(\mathrm{SiO}_{2}<54 \mathrm{wt} . \%, \mathrm{Mg} \#>0.65\right)$ played a relatively minor role in creating the compositional diversity of the Old Shiveluch magmas compared to the Young Shiveluch ones. The pronounced change in the Shiveluch magma compositions could have been related to adjustments of the magma plumbing system beneath Old Shiveluch following the large scale sector collapse in the Late Pleistocene that enabled a common mixing of evolved and primitive magmas on the later, Holocene stage of the volcano evolution.
\end{abstract}

(c) 2012 Elsevier B.V. All rights reserved.

\section{Introduction}

Shiveluch volcanic massif is located at the junction of the KurileKamchatka and Aleutian island arcs (Fig. 1a) and is considered to be one of the largest and most active volcanoes on the Kamchatka Peninsula. The volcanic massif has a number of unique features, which attract attention of researchers. Shiveluch volcano has a volume of $1000 \mathrm{~km}^{3}$ and has no analogs with respect to the amount of erupted andesites among all Quaternary volcanoes of Kamchatka (Melekestsev et al., 1991). The Holocene activity of the volcano was related to the Young Shiveluch eruptive center and characterized by numerous Plinian eruptions alternating with lava domes growth. The volume of the erupted

\footnotetext{
* Corresponding author.

E-mail address: n_gorbach@mail.ru (N. Gorbach).
}

products and the frequency of eruptions make this volcano the most active explosive center in Kamchatka (Ponomareva et al., 2007). Magnesian andesites are typical for this volcano and do not occur at other active volcanoes in Kamchatka (Volynets, 1994). A combination of a peculiar geodynamic setting and the abundance of magnesian andesites with adakitic signature on the recent stage of the Shiveluch Volcano evolution gave rise to the hypothesis that the volcano is fed by melting products of the Pacific plate subducted beneath northern Kamchatka (Yogodzinksi et al., 2001; Churikova et al., 2001; Münker et al., 2001). A further development of the hypothesis is presented by Ferlito (2011) who suggested that Shiveluch Volcano is probably located above two distinct slabs with different subduction angle and pressure/ temperature paths which are responsible for distinctive compositions of basaltic andesites erupted in the pre-caldera phase and the present-day magnesian andesites. Alternative hypotheses to explain 


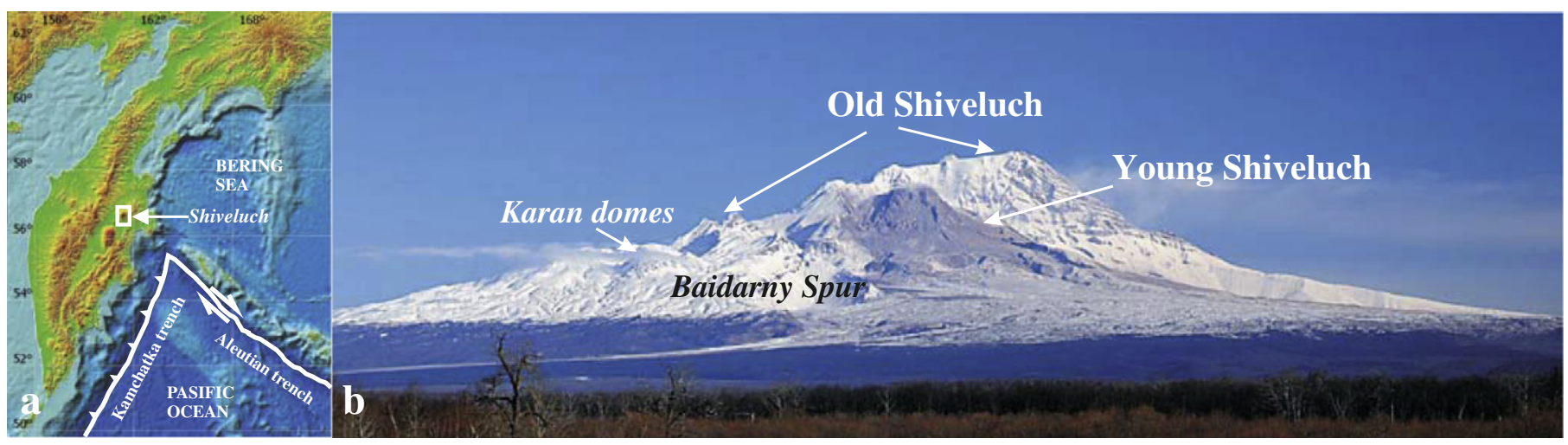

Fig. 1. Shiveluch volcanic massif: a - regional tectonic setting; and b - major structural units and geographic landmarks.

the unusual composition of Shiveluch rocks include an extensive interaction of magmas with lower crust (Melekestsev et al., 1991), a highly depleted mantle harzburgitic source (Volynets et al., 1999), or low temperature melting conditions in the mantle wedge beneath Shiveluch, which favored production of high-Si parental melts under hydrous melting conditions and/or predominant melting of fertile lithologies (pyroxenites) in the mantle wedge under fluid flux from the subducting plate (Portnyagin et al., 2007; Portnyagin and Manea, 2008; Portnyagin et al., 2009; Nikulin et al., 2012).

Shiveluch edifice (Fig. 1b) includes extinct Old Shiveluch volcano, which was partially destroyed by a large scale sector collapse, and the Young Shiveluch eruptive center, which has been active throughout the Holocene (Melekestsev et al., 1991). Whereas data on the eruptive history, petrology and geochemistry of Young Shiveluch are relatively abundant (Volynets et al., 1997; Dirksen et al., 2006; Ponomareva et al., 2007; Portnyagin et al., 2007; Gorbach and Portnyagin, 2011 and references therein), the history and composition of the Late Pleistocene Old Shiveluch volcano are known very fragmentary (Menyailov, 1955; Melekestsev et al., 1991; Volynets et al., 1999; Ferlito, 2011).

In this paper we present the results of a systematic geological and geochemical investigation of Old Shiveluch volcano. This research is aimed at filling the gap in knowledge of the structure, eruptive history and whole-rock composition of the volcano and allows recognizing some compositional changes of the erupted Shiveluch magmas since the Late Pleistocene.

\section{Shiveluch volcanic massif}

The Shiveluch volcanic massif covers an area of more than $1000 \mathrm{~km}^{2}$ in the northern part of the Central Kamchatka Depression (Fig. 1a). The relationships of Shiveluch volcanics with glacial deposits suggest the onset of the Shiveluch volcanic activity at 60-70 ka BP (Melekestsev et al., 1991). Shiveluch edifice comprises two main elements: Late Pleistocene Old Shiveluch and Holocene Young Shiveluch (Fig. 1b; Melekestsev et al., 1991). Old Shiveluch is a $3283 \mathrm{~m}$ high cone, cut by deep glacial valleys. It hosts a number of flank lava domes of different age, such as Late Pleistocene Semkorok domes in the southeast foot, Nordic domes on the northern flank and the Holocene Karan domes on the western slope (Fig. 3). The south-western sector of Old Shiveluch (also known as the Baidarny Spur, Fig. 1b) is believed to comprise many monogenetic volcanic centers (Volynets et al., 1999). The southern sector of the volcanic edifice was destroyed by a large-scale sector collapse which likely took place shortly before the Last Glacial Maximum (23-24 ka BP, Melekestsev et al., 1991). Alternatively, Belousov et al. (1999) suggested a younger age of this event $(\sim 10 \mathrm{ka} B P)$ based on the freshness of the Main Summit (3283 m.s.l, Fig. 3) upper lava flows. Based on field study and radiocarbon dating of debris avalanche deposits on the southern and south-eastern Shiveluch slopes, Pevzner et al.
(2012) have suggested that the destruction of the southern sector of the Old Shiveluch could have occurred in at least three stages with the earliest of these events dated at 15.8-16 ka cal BP (Pevzner et al., 2012). The Holocene eruptive center Young Shiveluch is located in the northwestern part of the collapse crater. Young Shiveluch had at least 60 large eruptions $\left(>0.5 \mathrm{~km}^{3}\right)$ in the Holocene, which resulted in the deposition of ignimbrite, ash fall and debris avalanche units and associated lahar deposits (Ponomareva et al., 2007).

Previous works considering the Old Shiveluch geology (Menyailov, 1955; Lopatin et al., 1979; Ermakov, 1985; Melekestsev et al., 1991) note that the edifice is composed by thick package of pyroclastic deposits, which are covered by numerous andesite and basaltic andesite lava flows. Based on new field mapping (Figs. 2 and 3 ) and geochemical analyses, we attempted to shed further light on the structure and rock compositions of the initial phase of the Old Shiveluch volcanic activity and the overlying lava complex.

\section{Geological structure of Old Shiveluch}

\subsection{Initial pyroclastic deposits}

Thick pyroclastic deposits, which form the base of the Old Shiveluch edifice (Fig. 3), are represented by weakly stratified agglomerate and psephitic tuffs (Fig. 4). The bottom of these deposits is not exposed. The upper boundary (between the tuffs and lava complex) is clearly visible in all sectors except for the southern foot, covered by the Young Shiveluch pyroclastic deposits. In the eastern sector of the volcano, the lowermost lava flows cover pyroclastic deposits at elevations of $1750-2000 \mathrm{~m}$ (Fig. 4a). In the western sector, the lava flows appear much lower at $\sim 1000 \mathrm{~m}$. The thickness of the initial Old Shiveluch pyroclastic deposits reaches $1800-2000 \mathrm{~m}$ in the central part of the volcano, and declines downslope to 900$1000 \mathrm{~m}$ at a distance of $7 \mathrm{~km}$ from the summit and to $400-500 \mathrm{~m}$ at a distance of $10-12 \mathrm{~km}$. According to our new mapping results, the initial pyroclastic deposits cover an area of about $220 \mathrm{~km}^{2}$ and comprise at least $60 \%$ of the Old Shiveluch volcanic edifice.

The initial pyroclastic deposits are represented predominantly by gravelly or agglomerate tuffs. These deposits usually contain 40$60 \mathrm{vol} . \%$ of pink or gray andesitic lithic clasts which vary in size from $20 \mathrm{~cm}$ to about $1-2 \mathrm{~m}$ (Fig. $4 \mathrm{~b}$ ). The clasts and blocks are set in psephitic and coarse-grained weakly lithified matrix of similar composition. The deposits are mainly poorly sorted, but vertical grading of lithic fragments is sometimes observed. The textures and grain-size distribution suggest that the deposits represent block-and-ash flow tuffs alternating with fall deposits.

The upper part of the initial pyroclastic deposits is best exposed in the Main Summit cliff (Fig. 4a). Here the section is dominated by lithoclastic psephitic andesitic tuffs with rare beds of basaltic andesite scoria (Fig. 4c). In the south-eastern sector of Old Shiveluch, the upper 


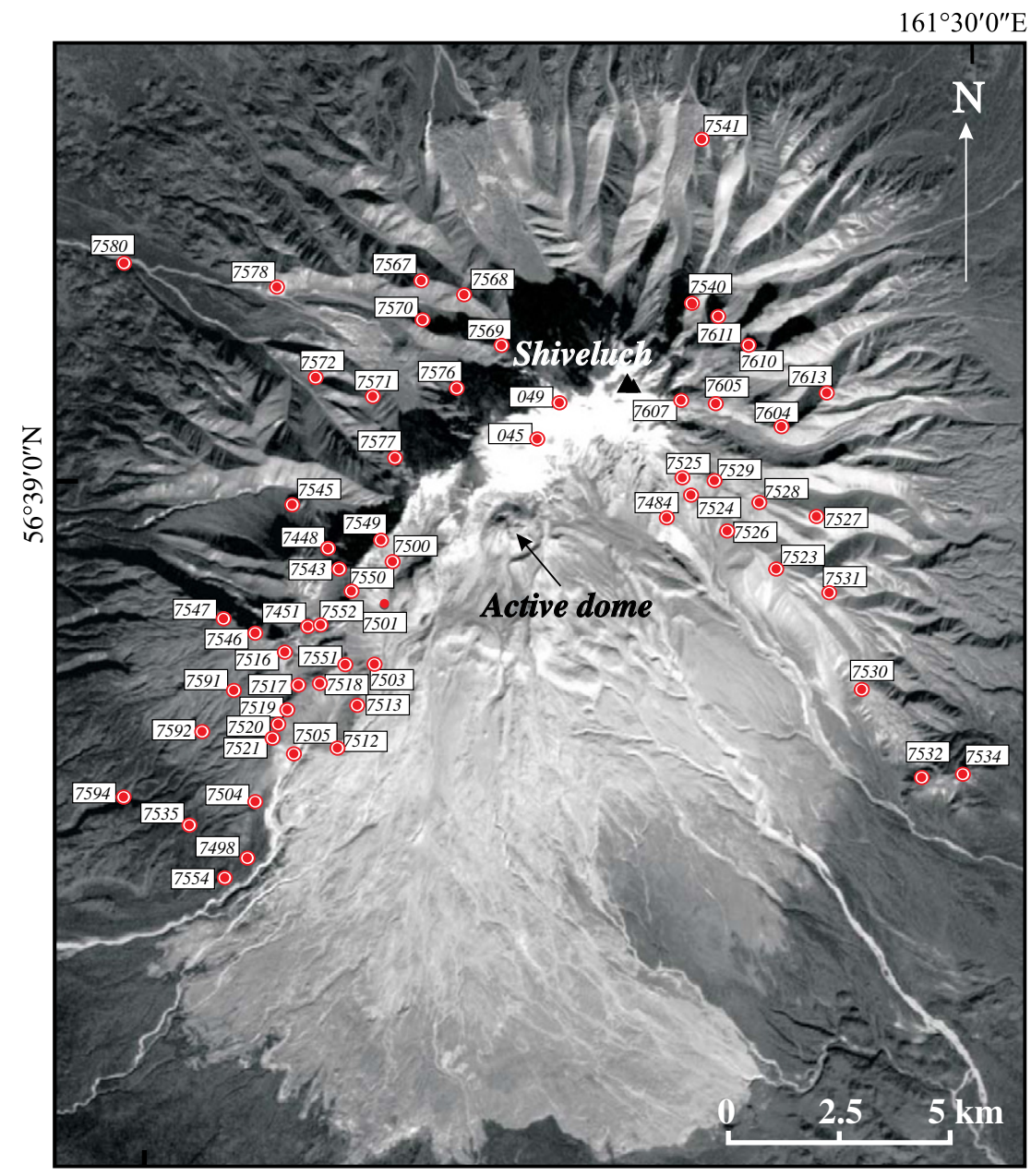

$161^{\circ} 10^{\prime} 0^{\prime \prime} \mathrm{E}$

Fig. 2. Scheme of sampling and observations points within Old Shiveluch edifice.

part of the initial pyroclastic deposits hosts several lava flows (Fig. 4d) of olivine-clinopyroxene-plagioclase basaltic andesites. These lavas cover the ridges between the valleys of the Dry Ilchinets and Topografichesky Creeks at the elevations of 1400-1600 m (Fig. 3). The lava flow thickness varies from 3 to $7 \mathrm{~m}$, and the length is up to $5 \mathrm{~km}$. Further east in the upper stream of the Topographichesky Creek, the olivine-clinopyroxene-plagioclase lava flows are overlaid by the Main Summit lava series.

\subsection{Old Shiveluch lava complex}

The Old Shiveluch lava complex comprises thick andesite and basaltic andesite lava flows. The sources of the lavas were at least four discrete vents whose position has been reconstructed based on field relationships of lava flows (Fig. 5). The Old Shiveluch Main Summit was constructed by a series of andesitic lava flows which are well exposed on the southern and eastern flanks (Fig. 6a). A large lava thickness and monocline bedding are the structural features of this lava series. The lowermost lava flows cover pyroclastic deposits of the initial stage near horizontally at the elevations of $1740-2000 \mathrm{~m}$. The flows have a near-uniform thickness which reaches $40-50 \mathrm{~m}$, and are sometimes interbedded with breccia and lenses of pyroclastic material. The upper lava flows overlie steeply the lower ones (at an angle up to $40^{\circ}$ for the apical flows) and have non-uniform thickness from $80 \mathrm{~m}$ in the vicinity of the Main Summit (Menyailov, 1955) to $20 \mathrm{~m}$ near the lava flow front. In the Old Shiveluch north-eastern sector, the upper lava flows are strongly eroded by modern glaciers. A $\sim 500 \mathrm{~m}$ deep crater with a diameter of $900-1000 \mathrm{~m}$ is located in the center of the summit ridge. The rocks surrounding the crater are strongly hydrothermally altered in a radius of $500 \mathrm{~m}$ from the crater rim. Periclinal lava bedding and signs of strong hydrothermal activity mark the position of the Central eruptive vent of the Old Shiveluch (Fig. 6b). The position of the Central eruptive vent is also marked by subvolcanic body (or cryptodome) of spherical shape and diameter of about $200 \mathrm{~m}$, which is located just below the Main Summit (Fig. 6b,c). In the summit cliff, it can be seen that the intrusion destroyed the primary bedding of the earlier lava flows and pyroclastic material. The layers of the lava flows and pyroclastics are displaced and broken. The deformation of the lava and pyroclastic deposits can be traced vertically for more than $1000 \mathrm{~m}$.

The structure of the lava series in the western sector of the old Shiveluch volcanic edifice is more complicated. Several eruptive vents were reconstructed there based on the lava flows dip and relationships in the preserved fragments (Figs. 5 and 7).

Similar to the Main Summit sector, the early lava flows cover initial pyroclastic deposits near-horizontally. Fragments of these flows occur at the elevations of $1000-1200 \mathrm{~m}$ in the upper streams of the Right Karina and Mutny Creeks. The thickest andesite lava flows are exposed in the 1st Lednikovy Creek in the northwestern part of the volcano. The dip of the lava flows indicates that the andesitic lavas originate from the Old Shiveluch Central vent.

The Western vent (Fig. 7a) was reconstructed $3 \mathrm{~km}$ to the west from the Central Old Shiveluch vent. The lava flows associated with the Western vent have periclinal dip and stretch out to the northwest, southwest and south. In the upper streams of the Mutny Creek and the Right and Left Karina rivers, the upper part of the section is 


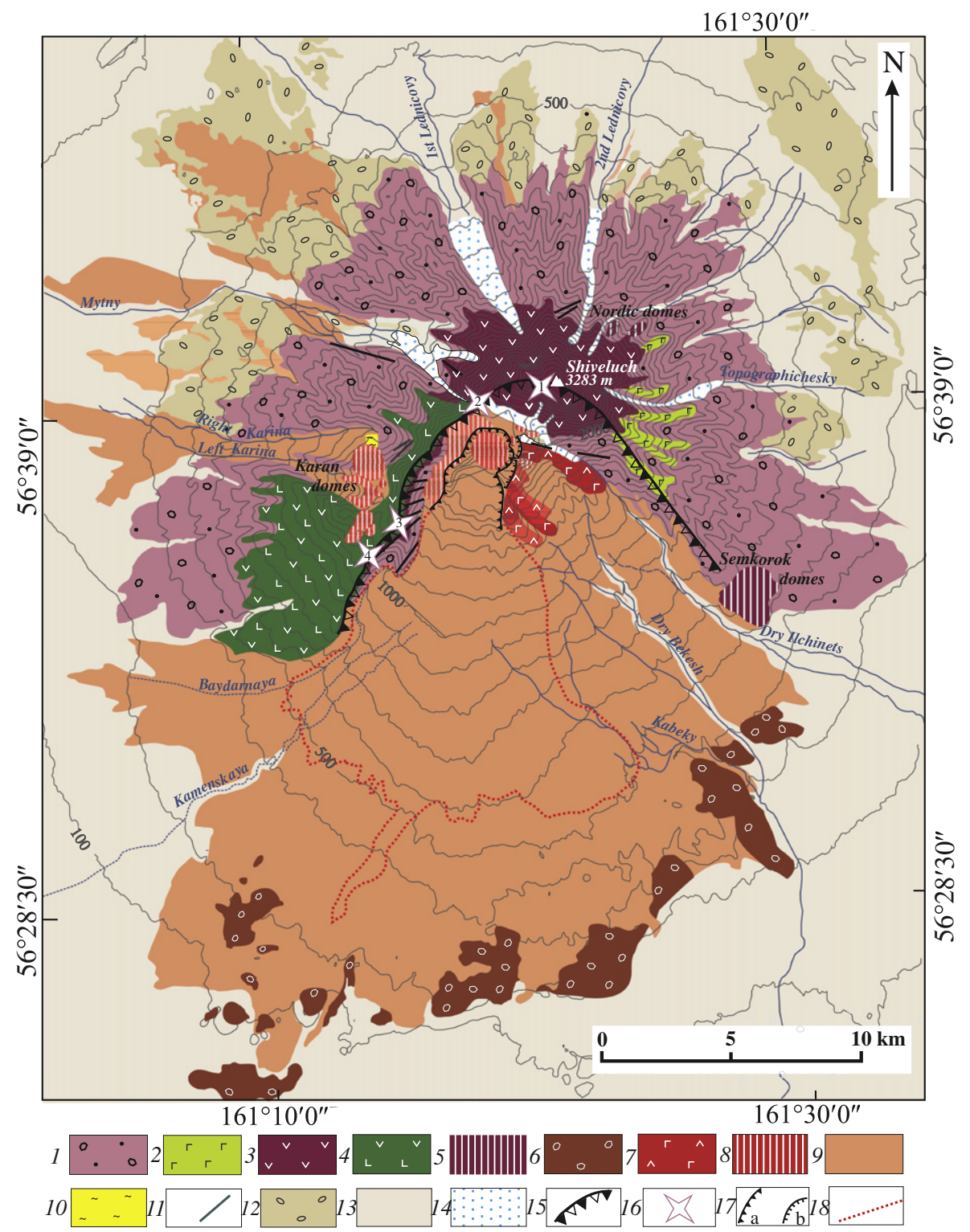

Fig. 3. Geological map of the Shiveluch volcanic massif compiled based on the 2006-2009 field observations and previously published data from Lopatin et al. (1979) and Melekestsev et al. (1991). The field of the Holocene proximal pyroclastic deposits is shown after Ponomareva et al. (2007). The field of proluvial deposits, moraines and expected Old Shiveluch sector collapse deposits allocated based on the interpretation of aerial photographs. Old Shiveluch (1-6): 1 - initial agglomerate tuffs of Hbl-Pl and Hbl-Px-Pl andesites, 2 - initial Ol-Cpx-Pl basaltic andesites, 3 - lavas of Hbl-Pl and Hbl-Px-Pl andesites, rarely lavas Px-Pl $\pm \mathrm{Hbl}$ basaltic andesites, $4-$ lavas of Px-Pl basaltic andesites, 5 extrusive lavas of $\mathrm{Hbl}-\mathrm{Pl} \pm \mathrm{Px}$ andesites, 6 - expected deposits of Old Shiveluch sector collapse. Young Shiveluch and Karan domes (7-9): 7 - lavas of Ol-Cpx-Pl $\pm \mathrm{Hbl}$ basaltic andesites and andesites, 8 - extrusive lavas of $\mathrm{Hbl}-\mathrm{Pl}$ and $\mathrm{Hbl}-\mathrm{Px}-\mathrm{Pl}$ andesites, rarely $\mathrm{Ol}-\mathrm{Hbl} \pm \mathrm{Pl}$ andesites, and 9 - proximal pyroclastic deposits. Other symbols (10-17): 10 - block of terrigenous rocks, 11 - dikes, 12 - moraines, 13 - proluvial and lahar deposits, 14 - modern glaciers, 15 - collapse crater rim, 16 - reconstructed Old Shiveluch vents ( 1 - Central, 2 - Western, 3 - Baidarny, 4 - Southern), 17 - crater of 1964 eruption, and 18 - boundary of 1964 eruption deposits.

significantly eroded by glaciers and partly destroyed by the subsequent Young Shiveluch activity. A well preserved fragment of the section occurs at the elevations of 2000-2400 m and consists of lavas of pyroxene-plagioclase basaltic andesites and pyroxene-hornblende andesites with thin scoria and breccia interbeds. The lava flows of the Western vent are overlapped by the lavas of more southern Baidarny vent (Fig. 7b).

The pyroxene-plagioclase basaltic andesite lava flows from the Baidarny vent cover the south-western slope of the Old Shiveluch and have a length of 5-6 km and descend to elevations of 600$700 \mathrm{~m}$. The Baidarny spur cliff exposes numerous dikes (Fig. 5). The northeast striking dikes intruded the pyroclastic deposits of the initial stage and the lower part of the Old Shiveluch lava complex. At least eight up to $20 \mathrm{~m}$ thick and up to $3 \mathrm{~km}$ long major dikes (some with offshoots) were recognized during the field mapping.
The fourth and smallest, Southern vent of the Old Shiveluch is located about $2.5 \mathrm{~km}$ to the southwest from the Baidarny Peak at the intersect of two dikes (Fig. 7c). It is a dome-shaped hill where several short lava flows of the total thickness up to $20 \mathrm{~m}$ are sourced. The Southern vent lavas overlap the Baidarny Peak lavas. This observation suggests the youngest age for the Southern vent of Old Shiveluch.

\section{Petrography of Old Shiveluch rocks}

All Old Shiveluch rocks are porphyritic and contain from 25 to $50 \%$ of phenocrysts by volume. Plagioclase phenocrysts are present in all rock varieties. The assemblage of mafic minerals is variable and allows distinguishing of five main types of rocks in the Old Shiveluch edifice: two-pyroxene \pm hornblende ( $\mathrm{Px}-\mathrm{Pl} \pm \mathrm{Hbl}$ ), pyroxene-hornblende-plagioclase (Px-Hbl-Pl), hornblende-plagioclase (Hbl-Pl) 

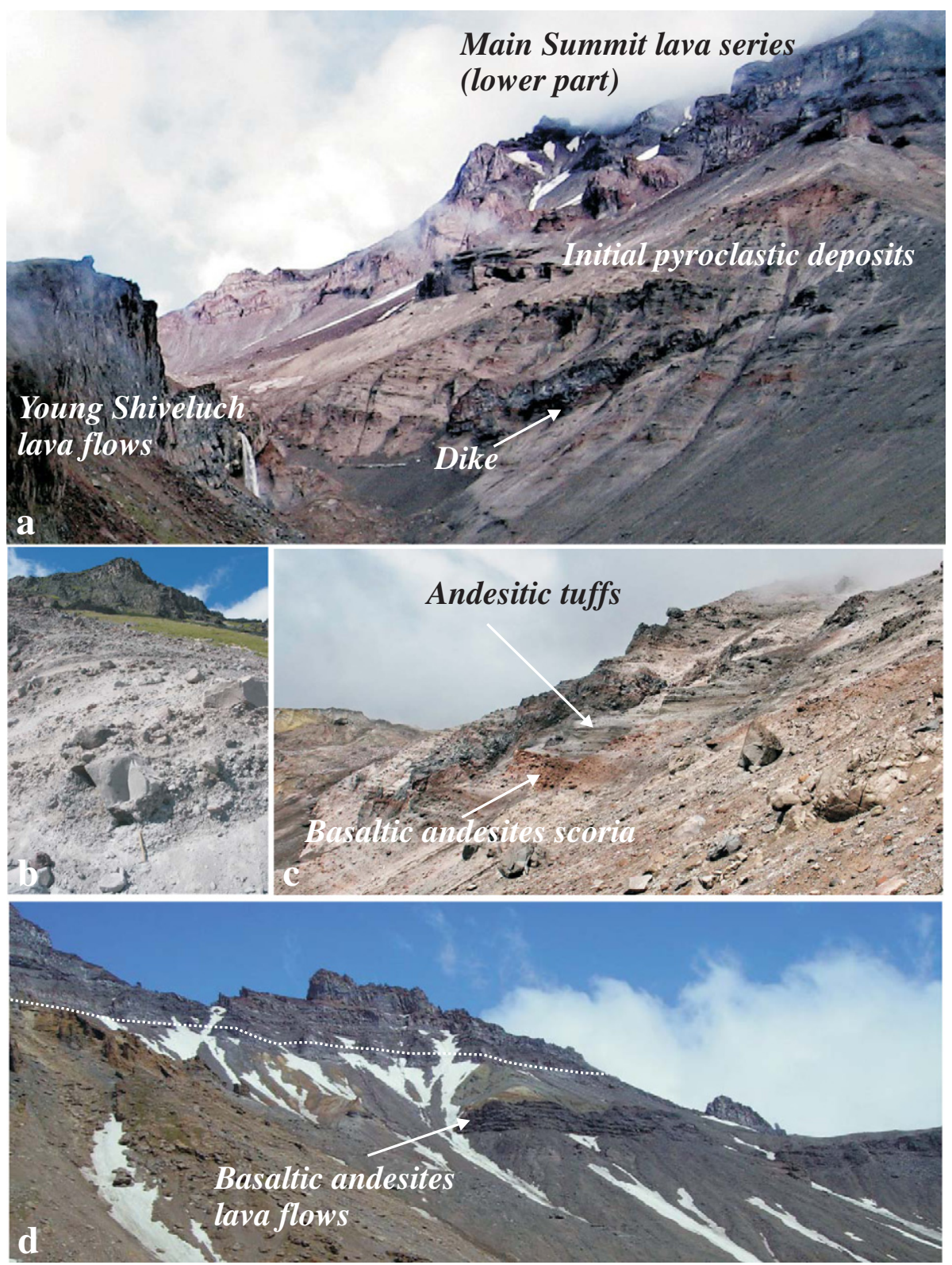

Fig. 4. Initial Old Shiveluch pyroclastic deposits: a - general view on the initial pyroclastic deposits in the central sector of the volcanic edifice. Apparent thickness of the deposits is about $800 \mathrm{~m}$; b - block-and-ash initial pyroclastic deposits in the Baidarny cliff; c - stratified andesitic lithoclastic tuffs interbedded with scoria of basaltic andesites; $\mathrm{d}$ - olivinepyroxene-plagioclase basaltic andesite lava flows in upper part of initial pyroclastic deposits (dotted line indicates the boundary between initial tuffs and lava complex).

andesites and olivine-clinopyroxene-plagioclase (Ol-Px-Pl) and pyroxene-plagioclase $(\mathrm{Px}-\mathrm{Pl})$ basaltic andesites.

$\mathrm{Px}-\mathrm{Pl} \pm \mathrm{Hbl}, \mathrm{Px}-\mathrm{Hbl}-\mathrm{Pl}$ and $\mathrm{Hbl}-\mathrm{Pl}$ andesites compose initial pyroclastic deposits, lava flows of the Central and Western vents and Nordic and Semkorok satellite lava domes. The andesites have similar petrographical features regardless of their structural affinity to either the initial pyroclastic or lava units. The andesites are strongly porphyritic rocks (25-50\% of phenocrysts) with predominant $\mathrm{Pl}\left(\mathrm{An}_{88-40}, 15-25 \%\right)$ and variable amounts of Cpx (2-7\%), Opx (2-4\%) and $\mathrm{Hbl}(0-12 \%)$. Fe-Ti oxide, apatite and sometimes crystobalite are present in the groundmass.

Small volume Ol-Cpx-Pl basaltic andesites appeared at the end of the initial pyroclastic phase of the Old Shiveluch activity. The rocks contain approximately equal amount of plagioclase (An 81-41, 12-15 vol.\%) and Fe-Mg mineral phenocrysts: Cpx (7-10 vol.\%), Ol (5-7 vol.\%), and Opx ( $\geq 2$ vol.\%). The occurrence of two generations of olivine phenocrysts, $\mathrm{FO}_{90-92}$ and $\mathrm{Fo}_{88-86}$ is a characteristic feature of these rocks. Some samples exhibit fluidal textures with similar orientation of large $\mathrm{Pl}$ phenocrysts and the presence of glass strips of color and optical properties different from the predominant groundmass glass. Some of these glass strips enclose large olivine and clinopyroxene phenocrysts. These petrographic features indicate increasing role of magma mixing toward the end of the initial pyroclastic phase of the Old Shiveluch activity.

The Px-Pl basaltic andesites associated with the Western, Baidarny and Southern eruptive centers are characterized by the sharp predominance of plagioclase phenocrysts (An 89-50, up to 20-30 vol.\%). Clino- and orthopyroxene are present in subordinate amounts of $7-10$ vol.\% and $3-5$ vol.\%, respectively. Olivine $\left(\mathrm{Fo}_{82-84}\right)$ is rare and usually surrounded by the reaction OPX-TiMt coronas. Relics of hornblende phenocrysts were also found. The groundmass is microlitic, intersertal or pilotaxitic and composed mainly by $\mathrm{Pl}$ and Px microlites, Fe-Ti oxides and volcanic glass.

\section{Whole rock geochemistry}

Sampling of the initial pyroclastic rocks was carried out in the western, northern and eastern sectors of the volcanic edifice at different 


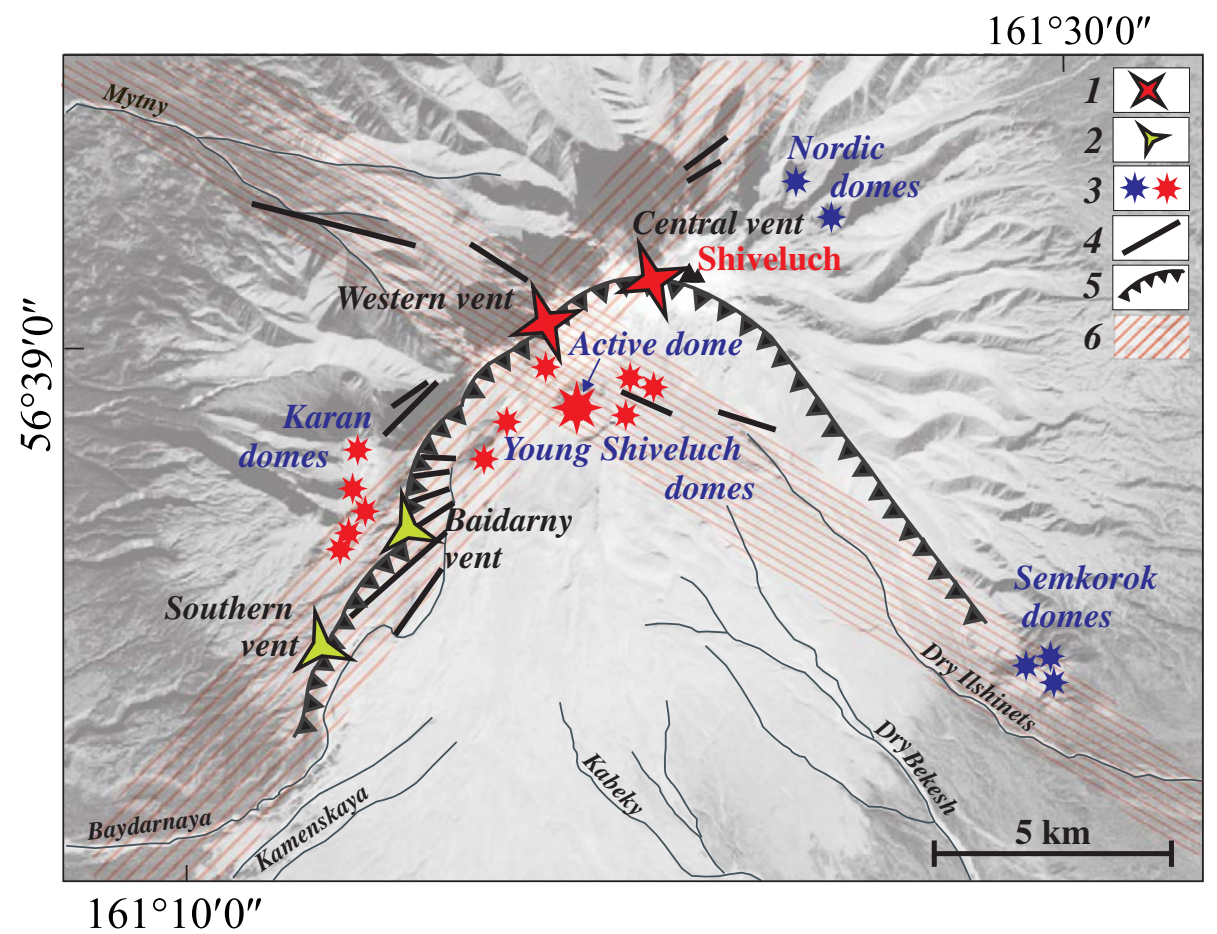

Fig. 5. Scheme of vents and dike distribution within Shiveluch volcanic massif. 1 - Old Shiveluch vents producing mainly andesitic lava; 2 - Old Shiveluch vents producing basaltic andesitic lava; 3 - Late Pleistocene (blue stars) and Holocene (red stars) evolved extrusive lava vents; 4 - dikes; 5 - collapse crater rim; and 6 - the assumed NE and WNW weakened zones.
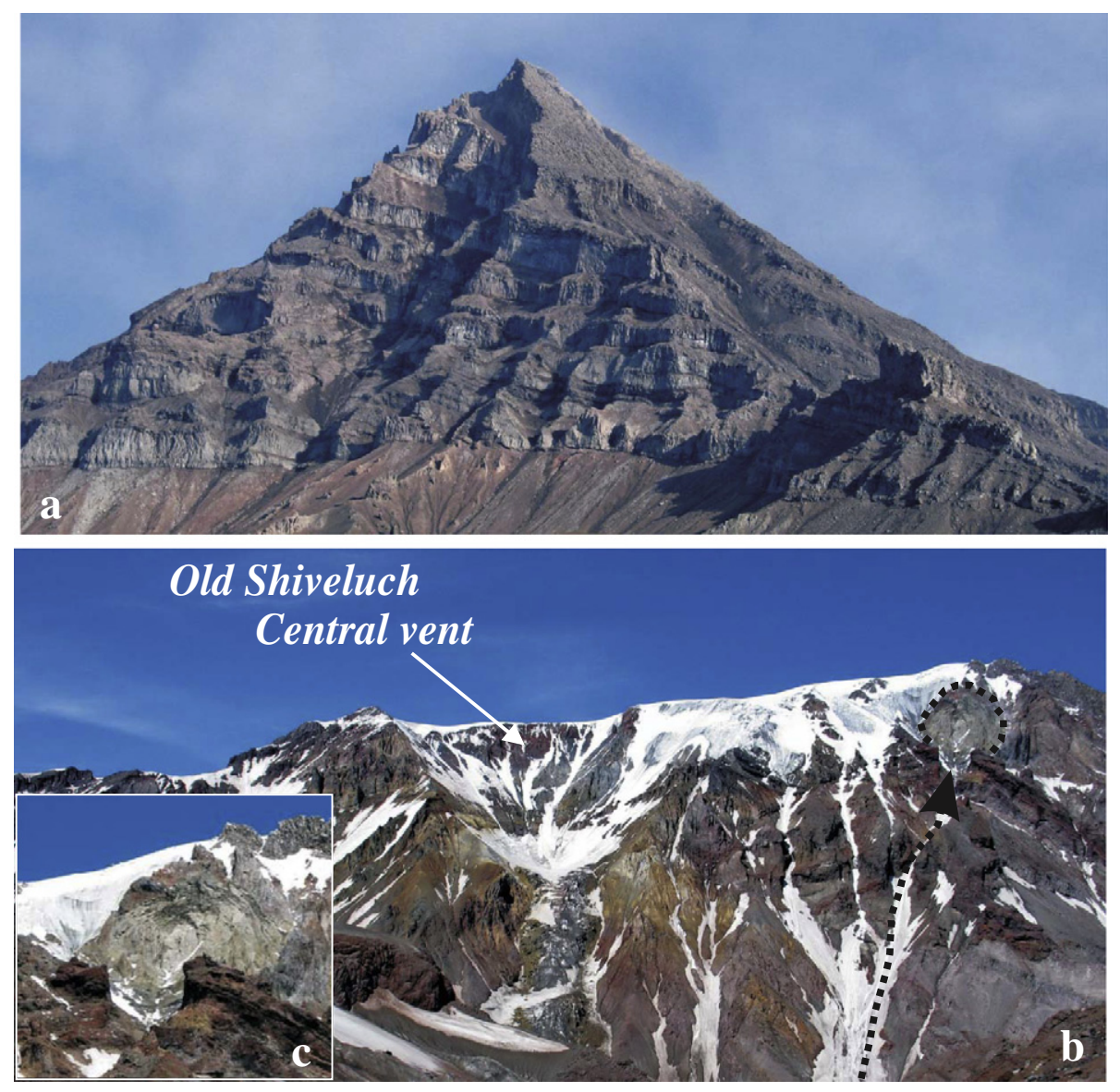

Fig. 6. Old Shiveluch Main Summit area: a - Main Summit lavas series (a view from south), b - the Central vent of Old Shiveluch and subvolcanic body (cryptodome) below the Main Summit (dotted line) and direction of its intrusion (dotted line with an arrow); and c - subvolcanic body (cryptodome) in largest scale. 

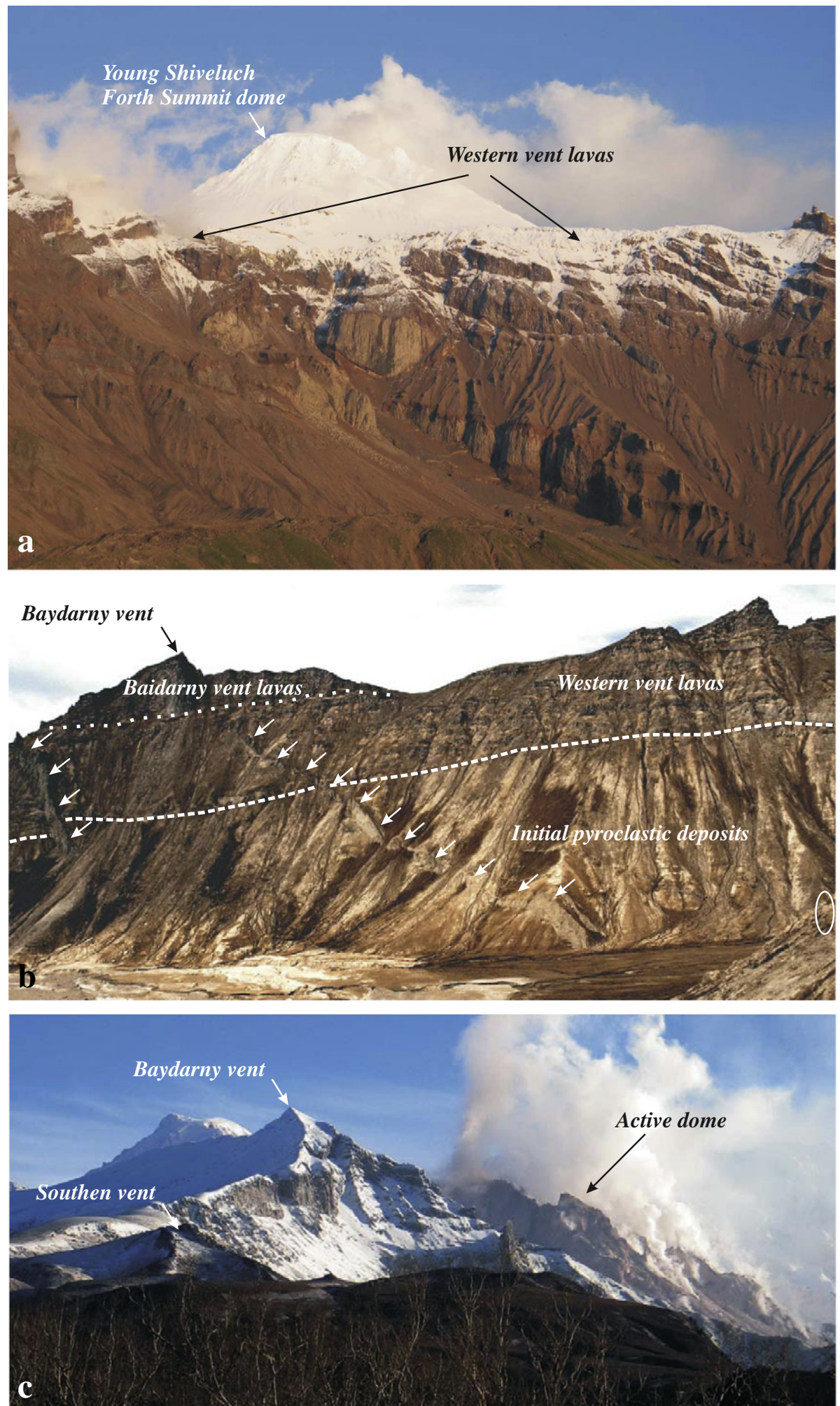

Fig. 7. Lavas in the western sector of Old Shiveluch: $a$ - position of the Western vent; $b$ - relationships between lavas of the Western and Baidarny vents (dotted line indicates the boundary between lavas of Western and Baidarny vents, the dashed line shows the boundary between Western vent lavas and initial pyroclastic deposits, dikes are marked by arrow); and $\mathrm{c}-$ position of the Baidarny and Southern vents.

elevations. The Old Shiveluch lava complex was sampled along the collapse crater rim and in the glacier craters in the upper streams of Mutny, Dry Ilchinets, 2nd Lednikovy and Topographichesky Creeks (Fig. 2). Fifty nine representative samples (Table 1 ) were selected to characterize geochemically all petrographic rock varieties of Old Shiveluch. Major elements ( $\mathrm{Si}, \mathrm{Ti}, \mathrm{Al}, \mathrm{Fe}, \mathrm{Mn}, \mathrm{Mg}, \mathrm{Ca}, \mathrm{Na}, \mathrm{K}$, and $\mathrm{P}$ ) and some trace elements ( $\mathrm{V}, \mathrm{Cr}, \mathrm{Co}, \mathrm{Ni}, \mathrm{Ga}, \mathrm{Sr}, \mathrm{Rb}, \mathrm{Ba}, \mathrm{Zr}, \mathrm{Nb}, \mathrm{Y}$, and $\mathrm{Zn}$ ) were analyzed in the rock samples by XRF technique Helmholtz Centre for Ocean Research Kiel (GEOMAR) Kiel, Germany. $\mathrm{H}_{2} \mathrm{O}$ and $\mathrm{CO}_{2}$ content were determined by infrared photometry. Details of the sample preparation and 
Table 1

The Old Shiveluch sample lists.

\begin{tabular}{|c|c|c|c|c|c|c|}
\hline$\#$ & Sample \# & Latitude & Longitude & Location & Material & Petrographic type \\
\hline \multicolumn{7}{|c|}{ Old Shiveluch initial phase } \\
\hline 1 & $7500-3$ & N56.62517 & E161.26912 & Upper Baidarnaya river & Block & $\mathrm{Hbl}-\mathrm{Pl}$ andesite \\
\hline 2 & 7505 & N56.58444 & E161.22164 & Upper Baidarnaya river & Block & Hbl-Px-Pl andesite \\
\hline 3 & $7541-1$ & N56.72112 & E161.38833 & Upper II Lednikovy Creek & Block & Hbl-Px-Pl andesite \\
\hline 4 & $7541-2$ & N56.72112 & E161.38833 & Upper II Lednikovy Creek & Block & Hbl-Pl andesite \\
\hline 5 & $7541-4$ & N56.72112 & E161.38833 & Upper II Lednikovy Creek & Block & Hbl-Px-Pl andesite \\
\hline 6 & 7531 & N56.61922 & E161.42044 & Upper Dry Ilchinets river & Lava flow & Hbl-Px-Pl andesite \\
\hline 7 & 7523 & N56.63461 & E161.39780 & Upper Dry Ilchinets river & Lava flow & Ol-Cpx-Pl basaltic andesite \\
\hline 8 & $7523-1$ & N56.63461 & E161.39780 & Upper Dry Ilchinets river & Lava flow & Ol-Cpx-Pl basaltic andesite \\
\hline 9 & $7526-2$ & N56.63798 & E161.39366 & Upper Dry Ilchinets river & Lava flow & Ol-Cpx-Pl basaltic andesite \\
\hline 10 & 7527 & N56.63611 & E161.42452 & Upper Dry Ilchinets river & Lava flow & Ol-Cpx-Pl basaltic andesite \\
\hline 11 & 7528 & N56.63893 & E161.41839 & Upper Dry Ilchinets river & Lava flow & Ol-Cpx-Pl basaltic andesite \\
\hline 12 & $7529-1$ & N56.64012 & E161.40132 & Upper Dry Ilchinets river & Lava flow & Ol-Cpx-Pl basaltic andesite \\
\hline 13 & $7529-2$ & N56.64012 & E161.40132 & Upper Dry Ilchinets river & Lava flow & Ol-Cpx-Pl basaltic andesite \\
\hline 14 & 7532 & N56.58091 & E161.45423 & Mount Semkorok & Extrusive lava & Hbl-Pl andesite \\
\hline
\end{tabular}

Old Shiveluch lava and dike complex

\begin{tabular}{|c|c|c|c|c|c|c|}
\hline 15 & 7524 & N56.63969 & E161.39522 & Main Summit cliff & Lava flow (CV) & Hbl-Px-Pl andesite \\
\hline 16 & $7524-2$ & N56.63969 & E161.39522 & Main Summit cliff & Lava flow (CV) & Hbl-Px-Pl andesite \\
\hline 17 & 7525 & N56.64058 & E161.39575 & Main Summit cliff & Lava flow (CV) & Hbl-Px-Pl andesite \\
\hline 18 & $7525-2$ & N56.64058 & E161.39575 & Main Summit cliff & Lava flow (CV) & Hbl-Px-Pl andesite \\
\hline 19 & $7525-3$ & N56.64058 & E161.39575 & Main Summit cliff & Lava flow (CV) & Hbl-Px-Pl andesite \\
\hline 20 & $7529-3$ & N56.64012 & E161.40132 & Main Summit cliff & Lava flow (CV) & Hbl-Px-Pl andesite \\
\hline 21 & 7540 & N56.68002 & E161.38488 & Northern flank & Lava flow (CV) & Hbl-Pl andesite \\
\hline 22 & 7451 & N56.61845 & E161.24022 & Upper Left Karina river & Lava flow (WV) & Px-Pl basaltic andesite \\
\hline 23 & 7503 & N56.57484 & E161.21417 & Upper Baidarnaya river & Lava flow (WV) & Hbl-Px-Pl andesite \\
\hline 24 & $7503-1$ & N56.57484 & E161.21417 & Upper Baidarnaya river & Lava flow (WV) & Px-Pl basaltic andesite \\
\hline 25 & $7543-1$ & N56. 62892 & E161.24812 & Upper Right Karina river & Lava flow (WV) & Px-Pl basaltic andesite \\
\hline 26 & $7543-3$ & N56. 62892 & E161.24812 & Upper Right Karina river & Lava flow (WV) & Px-Pl basaltic andesite \\
\hline 27 & 7544 & N56. 62742 & E161.25099 & Upper Right Karina river & Lava flow (WV) & Px-Pl basaltic andesite \\
\hline 28 & 7546 & N56. 61868 & E161.21943 & Upper Left Karina river & Lava flow (WV) & Px-Pl basaltic andesite \\
\hline 29 & 7548 & N56. 61787 & E161.24857 & Upper Left Karina river & Lava flow (WV) & Px-Pl basaltic andesite \\
\hline 30 & 7549 & N56. 62032 & E161.25520 & Upper Left Karina river & Lava flow (WV) & Px-Pl basaltic andesite \\
\hline 31 & $7550-2$ & N56. 61048 & E161.25280 & Upper Left Karina river & Lava flow (WV) & Px-Pl basaltic andesite \\
\hline 32 & $7550-3$ & N56. 61912 & E161.24363 & Upper Left Karina river & Lava flow (WV) & Hbl-Pl andesite \\
\hline 33 & 7567 & N56.68555 & E161.28500 & Upper Mytny Creek & Lava flow (WV) & Hbl-Px-Pl andesite \\
\hline 34 & $7568-1$ & N56.68638 & E161.28666 & Upper Mytny Creek & Lava flow (WV) & Hbl-Px-Pl andesite \\
\hline 35 & 7570 & N56.67583 & E161.29111 & Upper Mytny Creek & Lava flow (WV) & Hbl-Px-Pl andesite \\
\hline 36 & $7570-1$ & N56.67583 & E161.29111 & Upper Mytny Creek & Lava flow (WV) & Hbl-Px-Pl andesite \\
\hline 37 & 7577 & N56.66157 & E161.27050 & Upper Mytny Creek & Lava flow (WV) & Hbl-Px-Pl andesite \\
\hline 38 & 7504 & N56.58444 & E161.22164 & Baidarny spur & Lava flow (BV) & Px-Pl basaltic andesite \\
\hline 39 & 7513 & N56.59332 & E161.24956 & Baidarny spur & Lava flow (BV) & Px-Pl basaltic andesite \\
\hline 40 & 7516 & N56.60749 & E161.23049 & Baidarny spur & Lava flow (BV) & Px-Pl basaltic andesite \\
\hline 41 & 7517 & N56.60396 & E161.23037 & Baidarny spur & Lava flow (BV) & Px-Pl basaltic andesite \\
\hline 42 & 7518 & N56.60336 & E161.23374 & Baidarny spur & Lava flow (BV) & Px-Pl basaltic andesite \\
\hline 43 & 7551 & N56. 61872 & E161.25428 & Baidarny spur & Lava flow (BV) & Px-Pl basaltic andesite \\
\hline 44 & 7554 & N56. 56830 & E161.20697 & Baidarny spur & Lava flow (BV) & Px-Pl basaltic andesite \\
\hline 45 & 7519 & N56.60114 & E161.23008 & Baidarny spur & Lava flow (SV) & Px-Pl basaltic andesite \\
\hline 46 & 7521 & N56.59623 & E161.22697 & Baidarny spur & Lava flow (SV) & Px-Pl basaltic andesite \\
\hline 47 & K1-10A & N56. 59200 & E161.24800 & Middle Baidarnaya river & Lava flow (BV) & Px-Pl basaltic andesite \\
\hline 48 & $\mathrm{~K} 1-12$ & N56. 55000 & E161.18300 & Middle Baidarnaya river & Lava flow (BV) & Px-Pl basaltic andesite \\
\hline 49 & $\mathrm{~K} 1-14 \mathrm{~A}$ & N56. 55000 & E161.18300 & Middle Baidarnaya river & Lava flow (BV) & Px-Pl basaltic andesite \\
\hline 50 & K1-14B & N56. 55000 & E161.18300 & Middle Baidarnaya river & Lava flow (BV) & Px-Pl basaltic andesite \\
\hline 51 & 7501 & N56.61711 & E161.26802 & Upper Baidarnaya river & Dike & Hbl-Px-Pl andesite \\
\hline 52 & 7448 & N56.63233 & E161.24558 & Upper Right Karina river & Dike & Hbl andesite \\
\hline 53 & 7498 & N56.56429 & E161.20049 & Baidarny cliff & Dike & Px-Pl basaltic andesite \\
\hline 54 & $7500-2$ & N56.62517 & E161.26912 & Upper Baidarnaya river & Dike & Hbl-Px-Pl andesite \\
\hline 55 & $7500-4$ & N56.62517 & E161.26912 & Baidarny spur cliff & Dike & Hbl-Px-Pl andesite \\
\hline 56 & $7500-5$ & N56.62517 & E161.26912 & Upper Baidarnaya river & Blocks (dike?) & Ol-Cpx-Hbl basaltic andesite \\
\hline 57 & $7505-3$ & N56.58444 & E161.22164 & Baidarny spur cliff & Dike & Hbl-Px-Pl andesite \\
\hline 58 & 7512 & N56.59332 & E161.24956 & Baidarny spur cliff & Dike & Px-Pl basaltic andesite \\
\hline 59 & 7552 & N56. 61872 & E161.25428 & Upper Left Karina river & Dike & Px-Pl basaltic andesite \\
\hline
\end{tabular}

Note. For the Old Shiveluch lava complex the affiliation to discreet vents is shown (CV - Central vent; WV - Western vent; BV - Baidarny vent; SV - Southern vent).

analytical techniques are given in Portnyagin et al. (2005). The concentrations of major and selected trace elements in the Old Shiveluch rocks are reported in Table 2 .

The Old Shiveluch rocks vary in composition from basaltic andesites to andesites $\left(\mathrm{SiO}_{2}=53.5-63.8 \mathrm{wt} . \%\right)$ and belong to typical middle- $\mathrm{K}$ island-arc calc-alkaline series characterized by low $\mathrm{FeO}^{*} / \mathrm{MgO}$ (1.0-1.7) and moderate $\mathrm{K}_{2} \mathrm{O}$ contents $(0.87-1.67$ wt.\%) (e.g. Gill, 1981), which overlap with the composition of the Holocene Young Shiveluch lavas and pyroclastics (Fig. 8).
Based on petrographic features and composition, the Old Shiveluch rocks can be subdivided into three main groups (Figs. 9, 10). The most voluminous group of rocks is comprised by $\mathrm{Px}-\mathrm{Hbl}-\mathrm{Pl}$ and $\mathrm{Hbl}-\mathrm{Pl}$ magnesian andesites from the initial pyroclastic deposits, the Central and Western vent lava flows and the Nordic and Semkorok lava domes. These rocks are characterized by the following composition: $\mathrm{SiO}_{2}=$ 57.25-63.77, $\mathrm{TiO}_{2}=0.47-0.83, \mathrm{Al}_{2} \mathrm{O}_{3}=16.23-17.92, \mathrm{MgO}=2.8-5.06$, $\mathrm{K}_{2} \mathrm{O}=1.23-1.55$ (wt\%), $\mathrm{Cr}=45-102, \mathrm{Ni}=5-38(\mathrm{ppm})$, and $\mathrm{Mg} \#=$ $0.53-0.59$. The second group of rocks is represented by Px-Pl basaltic 
Table 2

Old Shiveluch whole rock major and trace element analyses.

\begin{tabular}{|c|c|c|c|c|c|c|c|c|c|c|c|c|c|c|c|c|c|c|c|c|c|c|c|c|c|c|}
\hline Sample \# & Mg\# & $\mathrm{SiO}_{2}$ & $\mathrm{TiO}_{2}$ & $\mathrm{Al}_{2} \mathrm{O}_{3}$ & $\mathrm{FeO}^{\mathrm{T}}$ & $\mathrm{MnO}$ & MgO & $\mathrm{CaO}$ & $\mathrm{Na}_{2} \mathrm{O}$ & $\mathrm{K}_{2} \mathrm{O}$ & $\mathrm{P}_{2} \mathrm{O}_{5}$ & LOI & $\mathrm{H}_{2} \mathrm{O}$ & $\mathrm{CO}_{2}$ & Total & Co & $\mathrm{Cr}$ & $\mathrm{Ni}$ & V & $\mathrm{Zn}$ & $\mathrm{Ga}$ & $\mathrm{Rb}$ & $\mathrm{Ba}$ & $\mathrm{Sr}$ & $\mathrm{Y}$ & $\mathrm{Zr}$ \\
\hline \multirow{2}{*}{\multicolumn{27}{|c|}{ Initial Old Shiveluch phase }} \\
\hline Initial pyro & & rocks & & & & & & & & & & & & & & & & & & & & & & & & \\
\hline $7500-3$ & 0.53 & 60.23 & 0.64 & 16.77 & 5.83 & 0.12 & 3.7 & 6.1 & 4.21 & 1.51 & 0.19 & & 0.34 & 0.02 & 99.66 & 23 & 59 & 11 & 167 & 67 & 20 & 25 & 404 & 492 & 17 & 113 \\
\hline 7505 & 0.53 & 57.81 & 0.7 & 17.18 & 6.30 & 0.14 & 4.03 & 7.13 & 4.18 & 1.37 & 0.22 & & 0.81 & 0.01 & 99.88 & 17 & 52 & 5 & 180 & 78 & 19 & 25 & 369 & 567 & 20 & 107 \\
\hline $7541-1$ & 0.53 & 57.48 & 0.72 & 17.05 & 6.44 & 0.13 & 4.11 & 7.1 & 4.31 & 1.45 & 0.24 & & 0.45 & 0.02 & 99.50 & 26 & 56 & 7 & 162 & 77 & 18 & 28 & 421 & 593 & 16 & 108 \\
\hline $7541-2$ & 0.55 & 60.58 & 0.54 & 16.89 & 4.81 & 0.11 & 3.25 & 5.81 & 4.78 & 1.41 & 0.18 & & 0.47 & 0.03 & 98.86 & 18 & 54 & 14 & 128 & 63 & 21 & 27 & 426 & 574 & 14 & 106 \\
\hline $7541-4$ & 0.53 & 58.64 & 0.68 & 7.25 & 6.22 & 0.13 & 3.94 & 6.98 & 4.28 & 1.5 & 0.25 & & 0.19 & 0.02 & 100.08 & 25 & 60 & 10 & 171 & 73 & 19 & 29 & 440 & 603 & 15 & 108 \\
\hline Initial lava & flows & & & & & & & & & & & & & & & & & & & & & & & & & \\
\hline 7531 & 0.53 & 57.94 & 59 & 7.56 & 60 & 12 & 59 & 5.97 & 4.43 & 1.33 & 0.32 & & 1.14 & 0.04 & 98.63 & 22 & 72 & 29 & 136 & 74 & 17 & 24 & 384 & 554 & 18 & 102 \\
\hline 7523 & 0.59 & 55.03 & 0.85 & 16.49 & 7.63 & 0.15 & 6.12 & 8.29 & 3.93 & 1.25 & 0.25 & & 0.04 & 0.01 & 100.04 & 34 & 175 & 35 & 223 & 79 & 19 & 22 & 304 & 523 & 20 & 94 \\
\hline 7523-1 & 0.64 & 53.92 & 0.87 & 16.15 & 7.64 & 14 & 52 & 37 & 4.11 & 1.18 & 0.25 & & 0.16 & 0.02 & 100.33 & 30 & 295 & 76 & 207 & 76 & 18 & 19 & 301 & 497 & 19 & 96 \\
\hline $7526-2$ & 0.62 & 54.63 & 0.76 & 15.27 & 8.03 & 0.15 & 7.36 & 9.12 & 3.1 & 1.18 & 0.23 & & 0.35 & 0.01 & 100.19 & 38 & 286 & 46 & 235 & 69 & 16 & 19 & 287 & 479 & 19 & 67 \\
\hline 7527 & 0.63 & 54.3 & 0.76 & 15.1 & 7.95 & 16 & 7.48 & 6 & 3.11 & 1.18 & 0.24 & & 0.56 & 0.06 & 100.06 & 33 & 315 & 512 & 240 & 68 & 14 & 23 & 272 & 477 & 17 & 72 \\
\hline 7528 & 0.62 & 54.85 & 0.76 & 15.25 & 8.10 & 0.15 & 7.46 & 9.18 & 3.13 & 1.18 & 0.24 & & 0.13 & 0.01 & 100.44 & 30 & 284 & 61 & 247 & 71 & 19 & 22 & 297 & 488 & 19 & 73 \\
\hline 7529-1 & 0.62 & 54.6 & 0.85 & 16.12 & 7.44 & 0.14 & 6.7 & 7.98 & 3.87 & 1.25 & 0.25 & & 0.29 & 0 & 99.49 & 32 & 236 & 73 & 201 & 78 & 20 & 23 & 324 & 506 & 18 & 98 \\
\hline $7529-2$ & 0.60 & 54.73 & 0.86 & 6.44 & 7.57 & 0.15 & 6.48 & 7.94 & 3.88 & 1.27 & 0.26 & & 0.21 & 0 & 99.79 & 32 & 218 & 69 & 216 & 79 & 19 & 22 & 305 & 523 & 21 & 95 \\
\hline Mount Sem & korok & & & & & & & & & & & & & & & & & & & & & & & & & \\
\hline 7532 & 0.57 & 63.77 & 0.47 & 16.43 & 4.47 & 0.09 & 3.33 & 5.1 & 4.63 & 1.26 & 0.15 & & 0.14 & 0.02 & 99.86 & 17 & 95 & 32 & 79 & 62 & 20 & 22 & 388 & 551 & 12 & 90 \\
\hline & & & & & & & & & & & & & & & & & & & & & & & & & & \\
\hline & t & n Sumn & $\mathrm{a}$ & flows) & & & & & & & & & & & & & & & & & & & & & & \\
\hline 7524 & 0.57 & 61.94 & 0.48 & 16.92 & 37 & 9 & 2 & 1 & 4. & 33 & 0.18 & & 12 & 0.09 & 9.10 & 15 & 44 & 6 & 112 & 50 & 20 & 27 & 408 & 548 & 14 & 99 \\
\hline 75 & 0.54 & 61.17 & 0.53 & 6.85 & 4.89 & 0.12 & 3.25 & 5.87 & 4.39 & 1.47 & 0.19 & & 0.77 & 0.02 & 99.52 & 22 & 62 & 12 & 122 & 61 & 17 & 29 & 431 & 542 & 13 & 105 \\
\hline 752 & 0.55 & 60.84 & 0.59 & 17.11 & 5.28 & 0.12 & 3.6 & 6.19 & 4.56 & 1.54 & 0.2 & & 0.12 & 0.01 & 100.16 & 17 & 55 & 10 & 146 & 69 & 19 & 29 & 442 & 533 & 17 & 105 \\
\hline $5-2$ & 0.55 & 60.78 & 0.58 & 17.01 & 5.22 & 0.12 & 3.56 & 6.1 & 4.35 & 1.55 & 0.2 & & 2 & 0.01 & 99.90 & 22 & 50 & 7 & 148 & 72 & 18 & 29 & 427 & 5 & 16 & 109 \\
\hline 75 & 0.54 & 57.73 & 0.73 & 17.05 & 6.87 & 0.14 & 4.48 & 7.27 & 4.0 & 1.39 & 0.21 & & & 0.01 & 100.11 & 2 & 74 & 16 & 195 & 77 & 20 & 23 & 370 & 5 & 20 & 99 \\
\hline-3 & 0.53 & 57.42 & 0 & 21 & 6.82 & 0.14 & 4.3 & 7.41 & 4. & 1. & 0.22 & & 3 & 0.01 & 95 & 23 & 69 & 10 & 195 & 77 & 20 & 24 & 357 & 560 & 19 & 95 \\
\hline 7540 & 0.53 & 62.56 & 0.49 & 16.87 & 4.43 & 0.11 & 2.8 & 5.56 & 4.6 & 1.48 & 0.1 & & & 0.03 & 100.42 & 16 & 46 & 5 & 111 & 58 & 18 & 27 & 414 & 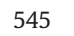 & 13 & 107 \\
\hline Western & & & & & & & & & & & & & & & & & & & & & & & & & & \\
\hline 7 & 0.52 & 0.14 & 0.93 & .53 & 77 & & 3 & 2 & 4. & 0.96 & 0. & & 29 & 0.03 & & 26 & 54 & 9 & 244 & 82 & 19 & 17 & 223 & 554 & 20 & 97 \\
\hline 7 & 0.55 & 57.53 & 0.8 & 17.07 & 6.78 & 013 & 4.61 & 7.38 & 4.38 & 1.21 & 0.19 & & 053 & 0.01 & 100.62 & 24 & 68 & 10 & 202 & 67 & 17 & 20 & 314 & 581 & 16 & 87 \\
\hline 7503-1 & 0.55 & 57.25 & 0.82 & 17.16 & 6.92 & 12 & 4.66 & 7.56 & 4.2 & 1.18 & 0.2 & & 13 & 0.03 & 100.28 & 30 & 71 & 12 & 209 & 68 & 18 & 20 & 303 & 586 & 14 & 89 \\
\hline 7 & 0.53 & 54.79 & 0.88 & 17.17 & 7.32 & 0.14 & 4.62 & 8.07 & 4 & 1.14 & 0.24 & & 0.13 & 0.00 & 98.50 & 28 & 2 & 4 & 235 & 73 & 21 & 27 & 295 & 568 & 20 & 97 \\
\hline 7543-3 & 0.54 & 54.12 & 0.9 & 17.03 & 7.48 & 0.14 & 4.88 & 8.27 & 3.98 & 1.07 & 0.23 & & & 0.03 & 6 & 28 & 64 & 3 & 228 & 67 & 21 & 20 & 324 & 538 & 19 & 102 \\
\hline 7 & 0.53 & 54.48 & 0.93 & 17.03 & 7.57 & 0.14 & 4.88 & 8.25 & 3 & 1.18 & 0.23 & & 0 & 0.02 & 76 & 31 & & t & & 75 & 21 & 24 & 35 & 5 & 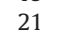 & 99 \\
\hline 75 & 0.55 & 53.94 & 0.88 & 16.58 & 7.89 & 0.16 & $\pi$ & 8.83 & 3. & 0.89 & 0.2 & & & 0.04 & 0 & 3 & 84 & 7 & 242 & 74 & 21 & 21 & 255 & 539 & 19 & 88 \\
\hline 75 & 0.53 & 54.25 & 0.92 & 16.71 & 7.82 & 015 & 5.01 & 8 & 3. & 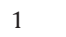 & 0.23 & & 25 & 0.0 & 43 & 2 & 60 & . & 234 & 76 & 23 & 19 & 268 & 546 & 21 & 98 \\
\hline & 0. & 54,42 & 0.92 & 16.93 & 7.7 & & & 8 & 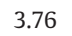 & 1 & 0.22 & & & 0.0 & & 3 & 55 & 2 & 242 & 78 & 21 & 21 & 280 & 5 & 21 & 98 \\
\hline & 0 & 54. & 0. & 16 & 7. & & & & 3. & 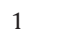 & 0 & & 1 & 0.02 & 98.33 & 32 & 50 & 8 & 234 & 74 & 18 & 21 & 248 & 549 & 21 & 101 \\
\hline & 0. & 61. & 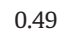 & 16.56 & 4. & 0 & 2 & 5 & 4. & 1.42 & 0.17 & & 0.64 & 0.02 & 98.40 & 16 & 73 & 11 & 99 & 61 & 21 & 32 & 416 & 573 & 16 & 112 \\
\hline $7567^{*}$ & 0.53 & 55.77 & 0.87 & 17.92 & 7.60 & 0.15 & 4.86 & 8.27 & 4.0 & 1.18 & 0.23 & 0. & & & 100.92 & & 49 & 17 & 251 & 97 & 19 & 18 & 296 & 558 & 21 & 94 \\
\hline & 055 & 59.70 & 060 & 1728 & 5 & & 3 & 6.73 & 4.4 & 1.30 & 0.19 & & & & 99.7 & & 66 & 21 & 153 & 75 & 19 & 22 & 369 & 553 & 17 & 110 \\
\hline 75 & 0.59 & 58.98 & 0.65 & 16.26 & 6.32 & 0.13 & 5.0 & 7. & 3. & 1.2 & 0.17 & & & & 100.2 & & 100 & 34 & 177 & 74 & 17 & 19 & 329 & 481 & 18 & 99 \\
\hline & 059 & 5 & $0 G 6$ & 53 & & 0 & 5. & 7.44 & 3. & 1.26 & 0.18 & 0 & & & 1 & & 102 & 33 & 8 & 75 & 18 & 21 & 337 & 479 & 17 & 100 \\
\hline $7577^{*}$ & 0.58 & 57.36 & 0.75 & 17.08 & 6.47 & 0.12 & 4.9 & 7.35 & 4.1 & 1.19 & 0.20 & 0.5 & & & 99.62 & & 80 & 38 & 186 & 71 & 19 & 19 & 306 & 537 & 19 & 108 \\
\hline Baidarny & ent & & & & & & & & & & & & & & & & & & & & & & & & & \\
\hline 7504 & 0.53 & 5 & & 7.38 & & & & & & & 0 & & & 0.02 & & 29 & 46 & 1 & 2 & 79 & 20 & 18 & 302 & 5 & 19 & 95 \\
\hline & & 55. & & & & & & & & & 0 & & & 0.00 & & & 70 & 11 & & 76 & 20 & 25 & & & 0 & 106 \\
\hline & 0 & 54. & $\mathrm{C}$ & 1. & I & & & & & & & & & 0.03 & 5 & J & 61 & 11 & 6 & 71 & 21 & 18 & 2 & 5 & 23 & 94 \\
\hline & 0.52 & 55. & 0.92 & 16.95 & 7.94 & 0. & 4. & 8 & 3. & 1.04 & 0.2 & & 0.30 & 0.03 & 100.08 & 3 & 58 & 7 & 243 & 79 & 20 & 18 & 276 & 544 & 21 & 97 \\
\hline & & 54 & & 17 & & & & & & & 0.2 & & & 0.01 & 99.32 & 3 & 67 & 26 & 302 & 76 & 20 & 19 & 304 & 568 & 17 & 89 \\
\hline & 0 & 54. & 0. & 16.78 & 7. & 0 & 4. & & 3. & 1. & 0.2 & & 6 & 0.0 & 98.39 & 31 & 60 & 16 & 234 & 83 & 21 & 18 & 267 & 541 & 24 & 99 \\
\hline & & 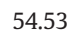 & 0 & & & & & & & & & & & 0. & & & $A 6$ & & & 76 & 22 & 24 & 297 & & 21 & 95 \\
\hline & 0 & 54. & 0.96 & 17.51 & 7.61 & 0. & 4. & & 4 & 1. & 0.2 & & 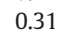 & 0.01 & 99.26 & 24 & 55 & 11 & 234 & 81 & 20 & 21 & 313 & 608 & 17 & 102 \\
\hline & & 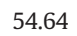 & & & & & & & 38 & & 0. & & & $0 . c$ & & 27 & 69 & 17 & & 77 & 21 & 17 & 250 & & & 98 \\
\hline & 0.57 & 55.27 & 0.87 & 16.95 & 7.31 & & 5.36 & 7.95 & 3. & 1.12 & 0.2 & & 0.10 & 0.00 & 9.18 & 23 & 99 & 25 & 220 & 73 & 18 & 21 & 288 & 524 & 16 & 91 \\
\hline & 0.58 & 5057 & 0.86 & 708 & 771 & & & 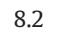 & & 1.08 & 0.23 & & & 0.01 & 98.86 & 2 & 03 & 26 & 223 & 74 & 20 & 19 & 287 & 556 & 17 & 93 \\
\hline South & ent & & & & & & & & & & & & & & & & & & & & & & & & & \\
\hline & 0.5 & 5 & & & & & & & & & 0 & & & 0 & & & & 1 & & 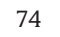 & 18 & & & & & 97 \\
\hline 7521 & 0.55 & 54.91 & 0.87 & 17.04 & 7.64 & 0.15 & 0.14 & 8.99 & 3.89 & 0.87 & 0.27 & & 0.26 & 0.02 & 100.05 & 32 & 86 & 15 & 220 & 70 & 20 & 14 & 253 & 544 & 20 & 91 \\
\hline & & & & & & & & & & & & & & & & & & & & & & & & & & \\
\hline 7448 & 0.60 & 57.66 & 0. & 16.52 & 5.76 & 0 & 4. & & 4. & 1.67 & 0.23 & & 0.14 & 0.02 & 99.32 & 30 & 69 & 13 & 163 & 72 & 18 & 20 & 466 & 1037 & 16 & 91 \\
\hline 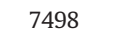 & 0.52 & 55. & 0.8 & 17. & & & & & 4. & 1.18 & 0.2 & & & 0.0 & & 2 & 47 & & 228 & 77 & 20 & 19 & 325 & 602 & 8 & 100 \\
\hline 7501 & & 59.32 & 0.64 & & 6.0 & & 4. & & 4. & & 0.18 & & & 0.02 & & 20 & 94 & 17 & 161 & 75 & 19 & 24 & 383 & 504 & 19 & 101 \\
\hline & 0.56 & 58. & 0.6 & 16.77 & 6.34 & & & & 4.1 & 1.33 & $0.1 \mathrm{C}$ & & & 0.01 & 5 & 23 & & & & & 19 & 23 & 322 & 51 & 7 & 98 \\
\hline $7500-4$ & 0.55 & 60.55 & 0. & 16.69 & 5.70 & 0. & 3.8 & & 4. & 1.53 & 0.19 & & & 0.03 & 100 & 19 & 77 & 11 & 156 & 67 & 18 & 29 & 442 & 513 & 18 & 106 \\
\hline $7500-5$ & 0.57 & 53.46 & 0.84 & 17.11 & 7.77 & 0.12 & 5.82 & 8. & 3.8 & 1.1 & 0.23 & & 0.88 & 0.03 & 99.48 & 31 & 75 & 29 & 220 & 73 & 20 & 18 & 283 & 562 & 19 & 88 \\
\hline $1000-3$ & 0.52 & 61.2 & & 17.36 & 4.98 & 0.1 & 3.0 & & 4.6 & 1. & 0.1 & & 0.16 & 0.02 & 99.91 & 15 & 45 & 3 & 144 & 61 & 21 & 25 & 394 & 598 & 12 & 108 \\
\hline 7512 & 0.52 & 55.16 & 0.96 & 17.63 & 7.61 & 0.12 & 4.6 & 8. & 4.03 & 1.17 & 0.26 & & 0.16 & 0.03 & 100.16 & 28 & 35 & & 236 & 80 & 19 & 20 & 317 & 617 & 20 & 95 \\
\hline 7552 & 0.53 & 53.78 & 0.93 & 16.94 & 7.85 & 0.15 & 4.96 & 8.44 & 3.7 & 0.91 & 0.24 & & 0.40 & 0.02 & 98.32 & 34 & 53 & 5 & 244 & 77 & 20 & 19 & 244 & 542 & 22 & 99 \\
\hline
\end{tabular}

Note. Major elements in wt.\%, trace elements in ppm. Mg\# refers to $\mathrm{MgO} /(\mathrm{MgO}+\mathrm{FeO})$ calculated on molar basic.

All analyses (except marked with an asterisk) were obtained by XRF technique at the Helmholtz Centre for Ocean Research Kiel (GEOMAR) Kiel, Germany. The sample \#s 7567*, $7568-1^{*}, 7570^{*}, 7570-1^{*}, 7577$ were analyzed by XRF at University of South Carolina, Columbia, USA. 

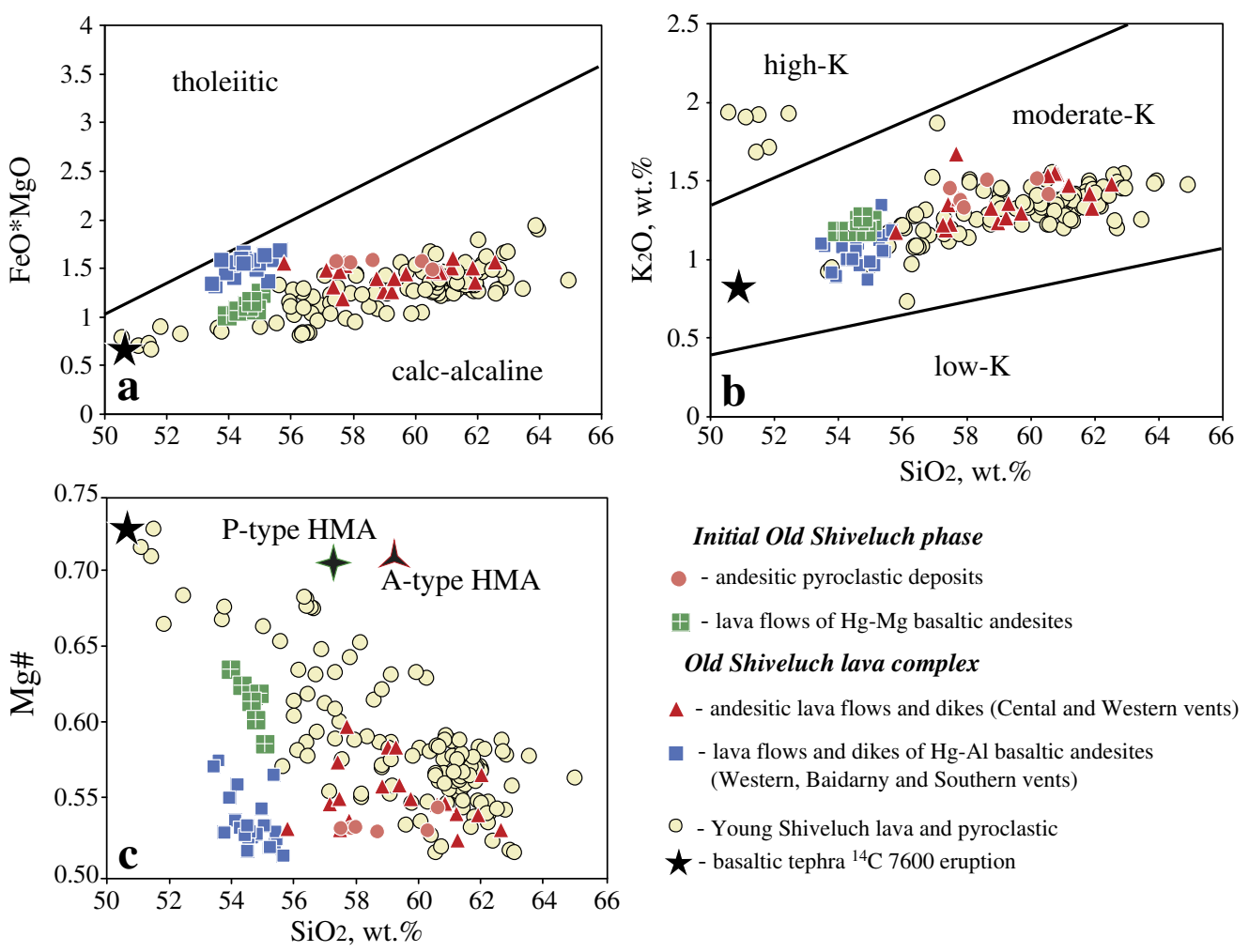

Fig. 8. Composition of Old Shiveluch rocks in $\mathrm{SiO}_{2}-\mathrm{FeO} / \mathrm{MgO}$ (a), $\mathrm{SiO}_{2}-\mathrm{K}_{2} \mathrm{O}$ (b) and $\mathrm{SiO}_{2}-\mathrm{Mg} \#$ (c) diagrams. The compositions of Young Shiveluch lavas and pyroclastics (Volynets et al., 1997; Ponomareva et al., 2007; Gorbach and Portnyagin, 2011), and A- and P-type primitive high-magnesian andesites (Yogodzinski et al., 1994, 1995) are shown for comparison.

andesites. These rocks are associated with the Western, Baidarny and Southern eruptive centers and have a narrow compositional range of major elements (in wt.\%) $\mathrm{SiO}_{2}=53.5-55.67, \mathrm{TiO}_{2}=0.84$ $0.99, \mathrm{Al}_{2} \mathrm{O}_{3}=16.58-17.51, \mathrm{MgO}=4.44-5.89$, and $\mathrm{K}_{2} \mathrm{O}=0.87-1.18$ and trace elements (in ppm), $\mathrm{Cr}=35-99, \mathrm{Ni}=2-26$, and $\mathrm{Mg} \#=0.52$ 0.56 . High-Mg basaltic andesites $(\mathrm{Mg} \#=0.59-0.64)$, which erupted at the end of the initial pyroclastic stage of the Old Shiveluch activity, have a small volume and local distribution. At a given $\mathrm{SiO}_{2}$ content, these rocks are slightly enriched in $\mathrm{MgO}$ (6.12-7.52 wt.\%), $\mathrm{K}_{2} \mathrm{O}$ (1.181.27 wt.\%), $\mathrm{Cr}$ (175-315 ppm), and $\mathrm{Ni}$ (35-76 ppm) and depleted in $\mathrm{Al}_{2} \mathrm{O}_{3}$ (15.1-16.49 wt.\%), and $\mathrm{TiO}_{2}$ (0.76-0.86 wt.\%) compared with high-Al basaltic andesites.

The compositions of all rocks from Old Shiveluch fall along relatively narrow evolutional trends (Fig. 9). The decrease in $\mathrm{MgO}$ is coupled with an increase in $\mathrm{SiO}_{2}, \mathrm{Al}_{2} \mathrm{O}_{3}, \mathrm{~K}_{2} \mathrm{O}$ and $\mathrm{Na}_{2} \mathrm{O}$ for all types of the Old Shiveluch rocks. $\mathrm{TiO}_{2}, \mathrm{FeO}^{*}, \mathrm{CaO}$ and $\mathrm{P}_{2} \mathrm{O}_{5}$ contents decrease slightly or remain nearly constant as $\mathrm{MgO}$ decreases from 7.5 to $\sim 5 \mathrm{wt} . \%$ and sharply decrease at lower MgO. Abundances of highly compatible trace elements $(\mathrm{Cr}$ and $\mathrm{Ni}$ ) decrease sharply with decrease of $\mathrm{MgO}$ (Fig. 10). The behavior of $\mathrm{V}$ and $\mathrm{Co}$ is similar to that of $\mathrm{FeO}^{*}$ and $\mathrm{TiO}_{2}$ and likely controlled by the appearance of $\mathrm{Fe}-\mathrm{Ti}$ oxides on the magma liquidus. Incompatible trace element concentrations (e.g. $\mathrm{Ba}, \mathrm{Rb}$ and $\mathrm{Zr}$ ) tend to increase with decreasing $\mathrm{MgO}$ and increasing $\mathrm{SiO}_{2}$ (Fig. 10). Ba and $\mathrm{Zr}$ contents are however somewhat scattered in the high-Mg and high-Al basaltic andesites that may suggest some compositional heterogeneity of parental magmas for these rocks.

It is noticeable that the most evolved and most primitive rocks from Old and Young Shiveluch, except for the Holocene high-K primitive basalts, have similar compositions. The evolutional trends of the rocks are however different for Old and Young Shiveluch. Whereas Old Shiveluch trends are strongly non-linear and likely reflect a sequential appearance of different mineral phases on the liquidus of fractionating magma, the trends of Young Shiveluch rocks, on the contrary, tend to be linear for most major and compatible ( $\mathrm{Ni}, \mathrm{Cr}$ ) trace elements plotted versus $\mathrm{MgO}$ or $\mathrm{SiO}_{2}$. In result, most of the Old Shiveluch rocks have lower $\mathrm{Mg} \#\left(=100 \mathrm{Mg} /\left(\mathrm{Mg}+\mathrm{Fe}^{*}\right)\right), \mathrm{Cr}, \mathrm{Ni}$, and $\mathrm{Ca}$, and higher $\mathrm{FeO}^{*}$ and $\mathrm{TiO}_{2}$ compared with the Young Shiveluch rocks (Fig. 8c) at a given $\mathrm{SiO}_{2}$ content.

Most of Old Shiveluch rocks have concentrations of $\mathrm{Al}_{2} \mathrm{O}_{3}, \mathrm{TiO}_{2}$ and $\mathrm{FeO}^{*}$ lower and $\mathrm{Na}_{2} \mathrm{O}$ higher than the Kliuchevskoi-Bezymianny rock series (Fig. 9). All Shiveluch rocks are significantly enriched in $\mathrm{Sr}$ and most of them depleted in Y compared with Kliuchevskoi and Bezymianny volcano rocks at similar $\mathrm{MgO}$ and $\mathrm{SiO}_{2}$. These new data implies different composition of parental magmas for KliuchevskoiBezymianny and Shiveluch rock series as has been suggested previously from the systematics of the most primitive rocks with Mg\#>0.6 along the Central Kamchatka Depression (e.g., Portnyagin et al., 2007).

\section{Discussion}

\subsection{Early Old Shiveluch eruptive activity}

Initial Old Shiveluch pyroclastic deposits have lithological features similar to those of the Young Shiveluch pyroclastic succession in the southern sector of the volcanic massif. The Holocene proximal deposits are represented by ash falls, pumice and block-and-ash pyroclastic density current deposits, debris avalanche and lahar deposits (Ponomareva et al., 2007). The composition, grain size distribution and stratification in the coarse agglomerate tuffs of Old Shiveluch are close to the characteristics of the modern block-and-ash pyroclastic flow deposits, agglomerate mantles of the lava domes and coarse tephra. The earlier and modern pyroclastic deposits have a similarly large area of distribution and near-horizontal bedding (Fig. 3).

The modern pyroclastic deposits are associated with the Young Shiveluch explosive activity, and growth, destruction and re-deposition of lava dome material. The lithological similarity of the Old Shiveluch 

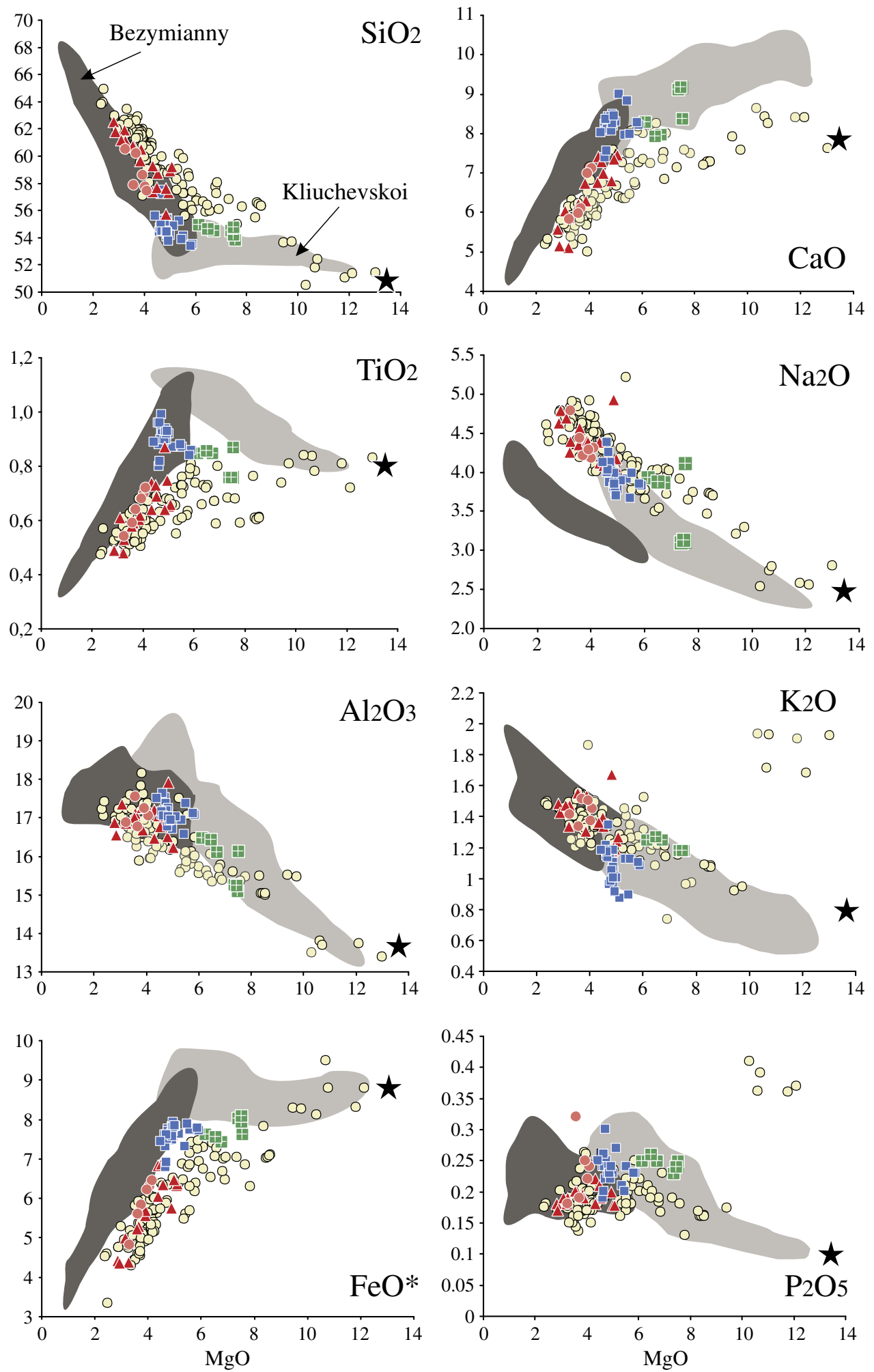

Initial Old Shiveluch phase

Old Shiveluch lava complex

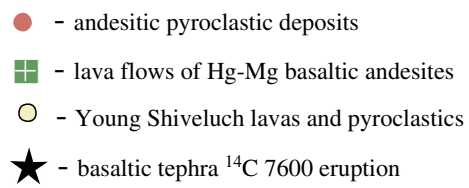

$\Delta$ - andesitic lava flows and dikes (Cental andWestern vents)

- lava flows and dikes of $\mathrm{Hg}-\mathrm{Al}$ basaltic andesites (Western, Baidarny and Southern vents)

Fig. 9. Variations of major element oxides in the Old Shiveluch rocks. Shadowed fields denote the composition of Klichevskoi (light gray field) and Bezymianny (dark gray field) volcano rocks (Portnyagin et al., 2007; Almeev et al., 2013) and illustrate expected trend of fractional crystallization of parental high-Mg basaltic magma at middle to upper crustal conditions. Linear trend of the Young Shiveluch rocks is readily explained by mixing of evolved and primitive magmas. 

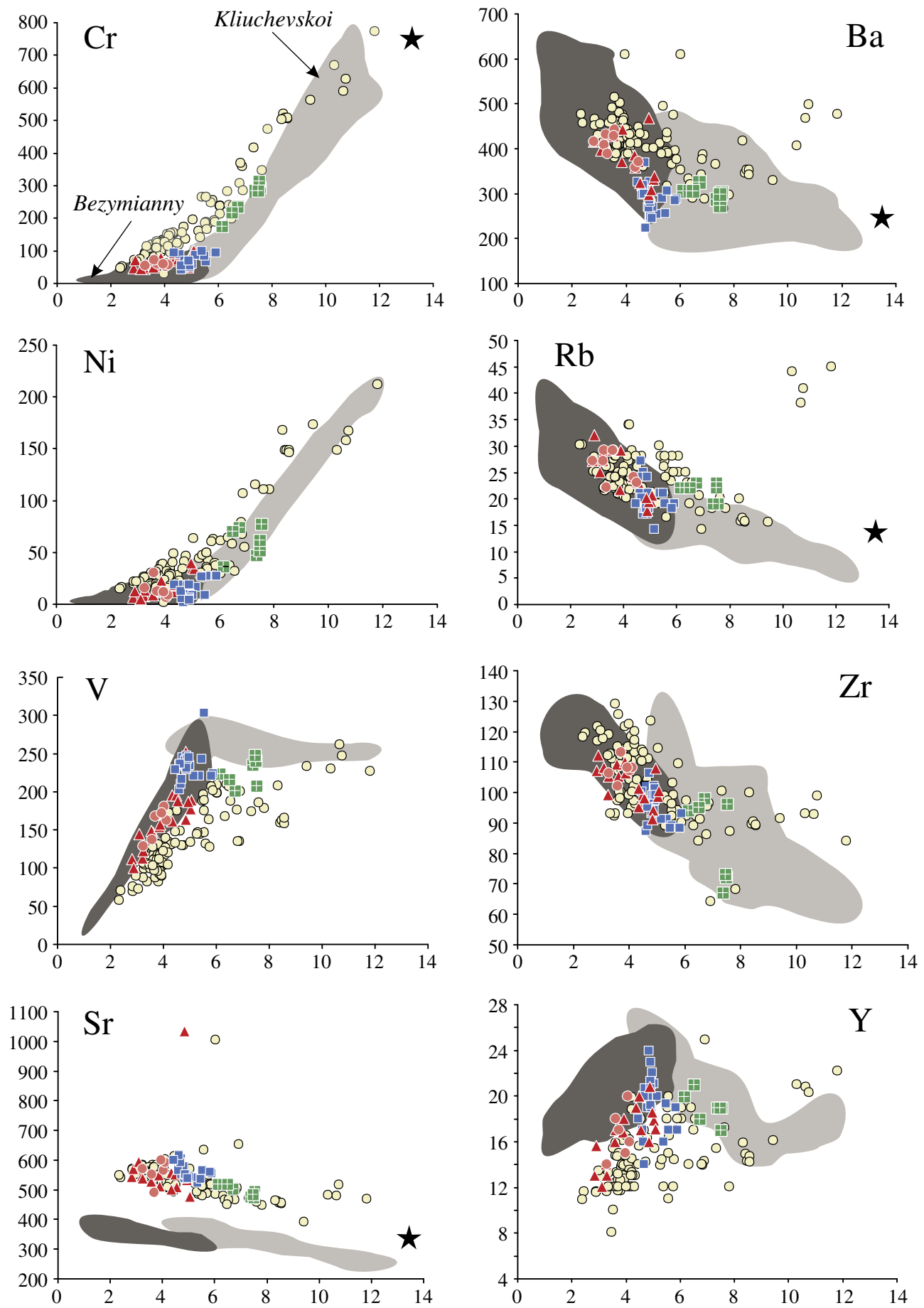

\section{Initial Old Shiveluch phase}

\section{Old Shiveluch lava complex}

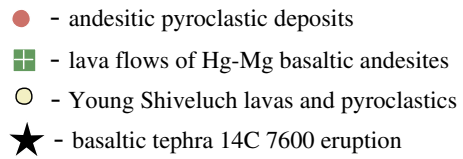

$\Delta$ - andesitic lava flows and dikes (Cental andWestern vents)

- lava flows and dikes of $\mathrm{Hg}-\mathrm{Al}$ basaltic andesites (Western, Baidarny and Southern vents)

Fig. 10. Variations of trace elements in the Old Shiveluch rocks. The fields denote the composition of Klyuchevskoy (light gray field) and Bezymianny (dark gray field) volcano rocks (Portnyagin et al., 2007; Almeev et al., 2013). Note the significantly higher Sr and lower Y content in both Old and Young Shiveluch magmas compared to Kliuchevskoi and Bezymianny that implies different compositions of parental magmas.

pyroclastic deposits and the modern deposits suggests that volcanic eruptions on the initial phase of the Old Shiveluch activity were similar to the Holocene activity and had extrusive-explosive style. It is thus plausible that emplacement of numerous lava domes was accompanied by strong explosive activity during the initial activity of Old Shiveluch. Nordic and Semkorok domes may be representative of the early extrusive bodies. However, the maximum thickness of the initial deposits is observed in the central sector of the Old Shiveluch edifice in the vicinity 
of the Main Summit. The dip of the stratified pyroclastic units is also predominantly directed away from the central sector. Therefore, the formation of the initial pyroclastic deposits had been most likely associated with a single eruptive center, similar to Young Shiveluch, but surpassing it significantly in size.

The apparent thickness of the initial pyroclastic deposits in the Old Shiveluch central sector greatly exceeds the thickness of the Holocene proximal pyroclastic deposits. The thickness of the Young and Old Shiveluch pyroclastic deposits is, however, similar at a distance of 7$12 \mathrm{~km}$ from the source and makes up a few hundred meters. The sharp increase of the initial pyroclastic deposits thickness in the Old Shiveluch central sector also is analogous to that in recent deposits. The rate of the debris and ash accumulation in the Young Shiveluch crater area is extremely high. For example, according to the field observations from December 2006 to September 2007, the material of hot avalanches and ash completely filled out the entire south-eastern sector of the modern crater, which was $>150$ m deep prior to the eruption. The intra-crater modern deposits, which form the agglomerate mantle of the growing dome, exhibit a rough stratification of poorly sorted debris, associated with hot avalanches and interbedded with coarse-grained ash layers. Thus, the coarse stratified deposits of the agglomerate mantles may have also been a part of the initial pyroclastic deposits and significantly increased its thickness in the central part of the volcano.

\subsection{Eruption sequence and volume proportions of the Old Shiveluch lavas}

The relations between lava flows of the Old Shiveluch lava complex indicate that they erupted from discrete vents which were different in terms of the erupted lava volume. The Old Shiveluch volcanic edifice is strongly destroyed (Fig. 11b), especially in the western sector. It is, therefore, very difficult to estimate precisely the volume proportions of lavas erupted from different vents. Nevertheless, taking into account the apparent thickness of the preserved lava sections, the most voluminous eruptions were likely associated with the Central and Western vents. The thickness of lavas related to these vents reaches more than $1000 \mathrm{~m}$ in a radius of $\sim 2 \mathrm{~km}$ from the Main Summit. Lavas of Px-Pl basaltic andesites and Px-Hbl-Pl andesites related to the Western vent are enclosed between typical andesitic lava flows of the Central vent (Fig. 11c), that suggests a simultaneous activity of the Central and Western vents. The Baidarny and Southern vents which produced exclusively Px-Pl basaltic andesites were formed later. The lavas of the Baidarny vent overlap those produced by the Western vent and underlie the Southern vent lavas (Fig. 11c). This implies that eruptive center of the Old Shiveluch migrated in the south-western direction over time. Based on the stratigraphic relationships of the lavas, the Southern vent is considered to be the youngest in the western sector of the Old Shiveluch volcanic edifice. The total thickness of lavas erupted from the Baidarny and Southern vents does not exceed $200 \mathrm{~m}$, although the Px-Pl basaltic andesites lavas occupy somewhat larger area in comparison with the andesites from the Central and Western vents. Thus apparent volume ratio of the Old Shiveluch andesitic and basaltic andesitic lavas is close to 1:0.75. Given the strong predominance of andesites during the initial pyroclastic stage, the volume ratio of andesites and basaltic andesites in the Old Shiveluch edifice can be estimated to be $4: 1$.

\subsection{The position of dikes in the Old Shiveluch edifice and prerequisites of the sector collapse}

It is known that radial dikes intrude into volcanic edifice due to pressure excess in magma chamber (Nakamura, 1977). Grouping of dikes in certain directions is less dependent on the magma chamber overpressure and is controlled by regional tectonic stresses (Nakamura, 1977; Acocella and Neri, 2009). The size and configuration of volcanic edifice and magma reservoir, and the edifice load also may have effect on the direction of the dike emplacement (e.g., Pinel and Jaupart, 2003; Simakin and Chassemi, 2009). Fig. 5 illustrates the distribution of dikes within the Old Shiveluch volcanic massif. Two directions predominate in the dike trending: northeastern $\left(40-50^{\circ}\right)$ and west-northwestern $\left(290-310^{\circ}\right)$. NE-trending dikes are exposed in the Baidarny Spur cliff. To the north of Baidarny Peak, a few dikes change direction to a well-defined radial character. WNW-trending dikes are younger and localized on the saddle between Young and Old Shiveluch and are traced in NW volcanic sector. We can assume that emplacement of the dikes deflected from the radial direction was confined to the NE and WNW-weakened zones (Fig. 5) which may reflect the regional tectonic stress. The dike distribution within the Shiveluch volcanic massif outlines the destroyed southern sector of the Old Shiveluch volcanic edifice. The reconstructed eruptive vents are also located on the perimeter of the collapse crater (Figs. 3 and 5). These structural features of the

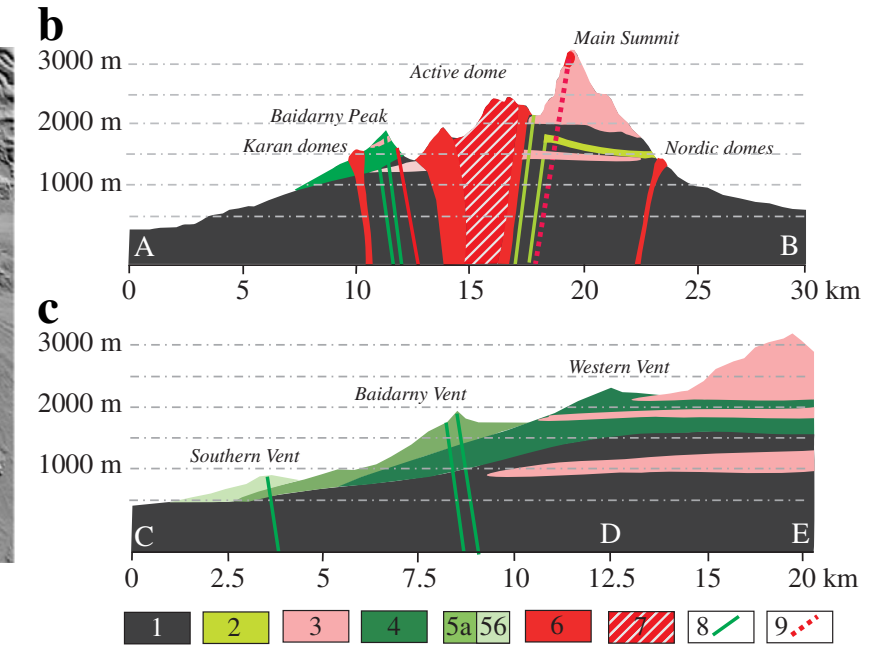

Fig. 11. Schematic sections across Shiveluch volcanic massif: a - position of the section; b - general section including the Young Shiveluch edifice; $c$ - section showing the lavas relation in western sector of Old Shiveluch edifice. 1 - initial pyroclastic deposits; 2 - lavas of high-Mg basaltic andesites erupted at the end of the Initial stage; 3 - andesites lavas of the Central and Western Old Shiveluch vent; 4 - lavas of high-Al basaltic andesites of the Western Old Shiveluch vent; 5 - lavas of high-Al basaltic andesites (5a - Baidarny vent; 5b - Southern vent); 6 - extrusive lavas; 7 - extrusive lavas of the modern dome; 8 - dikes; and 9 - direction of subvolcanic body (or cryptodome) intrusion. 
volcanic massif indicate that the collapse of the southern sector of Old Shiveluch could be pre-conditioned by the regional tectonic structure.

\subsection{The origin of temporal compositional variability of Shiveluch magmas}

The most evolved Holocene (Young Shiveluch) and Late Pleistocene (Old Shiveluch) rocks ( $\mathrm{SiO}_{2} \geq 60$ wt.\% and $\mathrm{MgO} \leq 4$ wt.\%) have similar compositions. The compositions of more primitive rocks are different. In particular, most of the Old Shiveluch rocks have lower $\mathrm{Mg} \#, \mathrm{MgO}, \mathrm{Cr}, \mathrm{Ni}$, and $\mathrm{CaO}$, and higher $\mathrm{Al}_{2} \mathrm{O}_{3}, \mathrm{FeO}^{*}$ and $\mathrm{TiO}_{2}$ compared with Young Shiveluch rocks (Fig. 8c) at a given $\mathrm{SiO}_{2}$. The trends of Old Shiveluch rocks are strongly non-linear with clearly pronounced turning points. The trends of Young Shiveluch rocks, on the contrary, tend to be linear for most major and compatible trace elements plotted versus $\mathrm{MgO}$ or $\mathrm{SiO}_{2}$.

One possible explanation for the compositional difference between Old and Young Shiveluch rocks could be different composition of parental magmas and their sources. In particular, Ferlito (2011) proposed that Baidarny Spur high- $\mathrm{Al}_{2} \mathrm{O}_{3}$ basaltic andesites, somewhat similar to the products of Kliuchevskoi and other volcanoes in Kamchatka, can be associated with partial melting of the mantle wedge hydrated by the subducting slab. The Young Shiveluch andesites, with high $\mathrm{Mg \# ,} \mathrm{Cr}$ and Ni signature, were interpreted to be primary slab melts which have assimilated peridotite in the overlying mantle wedge.

Old Shiveluch rocks and particularly basaltic andesites of Baidarny Spur have relatively low Mg\# $(<0.65)$ to be derived from the mantle wedge $(\mathrm{Mg} \#>0.70$ in order to be in equilibrium with mantle olivine of Fo $>90$ ). Thus, the magmas experienced significant crystal fractionation before eruption. High-Mg basaltic andesites and andesites of Young Shiveluch also have too low Mg\# compared to near primary magnesian andesites with $\mathrm{Mg \#}>0.7$, for example, those from the Komandorsky Block (A-type andesites, Yogodzinski et al., 1995) or from Piip Volcano (P-type andesites, Yogodzinski et al., 1994) in the Far-Western Aleutian Arc (Fig. 8c). Therefore high-Mg andesites of Young Shiveluch also are products of significant fractionation of primary magmas prior to eruption and cannot be interpreted as primary andesitic liquids. A careful petrological analysis is required to verify the interpretation of the compositional difference between Old and Young Shiveluch magmas as reflecting the difference in their magma sources. Indeed, Portnyagin et al. (2007) in their comprehensive study of primitive rocks of CKD found no apparent bimodality in the compositions of potential parental magmas of Shiveluch Volcano. An alternative hypothesis must be therefore tested that the substantial range of $\mathrm{SiO}_{2}$ content at moderately high $\mathrm{Mg} \#$ in Shiveluch magmas can be produced by fractionation of a single parental melt in the crust.

In order to assess the possible effects of crystallization on major element composition of Shiveluch magmas, we performed a numeric modeling of fractional crystallization of a potential primary Shiveluch magma. As a starting composition we chose the most primitive middle-K basaltic tephra (sample 90/122-2; Volynets et al. (1997)) erupted $7600{ }^{14} \mathrm{C}$ years ago. This sample has $\mathrm{MgO} \sim 13 \mathrm{wt} . \%, \mathrm{Mg} \#=$ 0.73, liquidus olivine with Fo-number of $92.3 \mathrm{~mol} \%$ and can well represent the composition of nearly unmodified mantle derived melt. Fractional crystallization of this basalt was simulated for initial $\mathrm{H}_{2} \mathrm{O}$ content 3 wt.\% (Portnyagin et al., 2007), oxygen fugacity of $\Delta \mathrm{QFM}=+2$ (Gorbach and Portnyagin, 2011) and pressures of 0.1 , 0.5 and $1 \mathrm{GPa}$, corresponding to upper, middle and lower crust beneath the Central Kamchatka Depression (e.g., Levin et al., 2002). The simulation of fractional crystallization was performed in Petrolog ver. 3.1.1.3 software (Danyushevsky and Plechov, 2011) by using models of Danyushevsky (2001) for determination of the liquidus temperatures and compositions of olivine, clinopyroxene and plagioclase, model of Ariskin and Barmina (1999) for Fe-oxides, and model of Kress and Carmichael (1988) for $\mathrm{Fe}^{2+} / \mathrm{Fe}^{3+}$ ratio in melt.

The results of our simulations are illustrated in Fig. 12. These data show that the compositions of high-Al basaltic andesites of Old
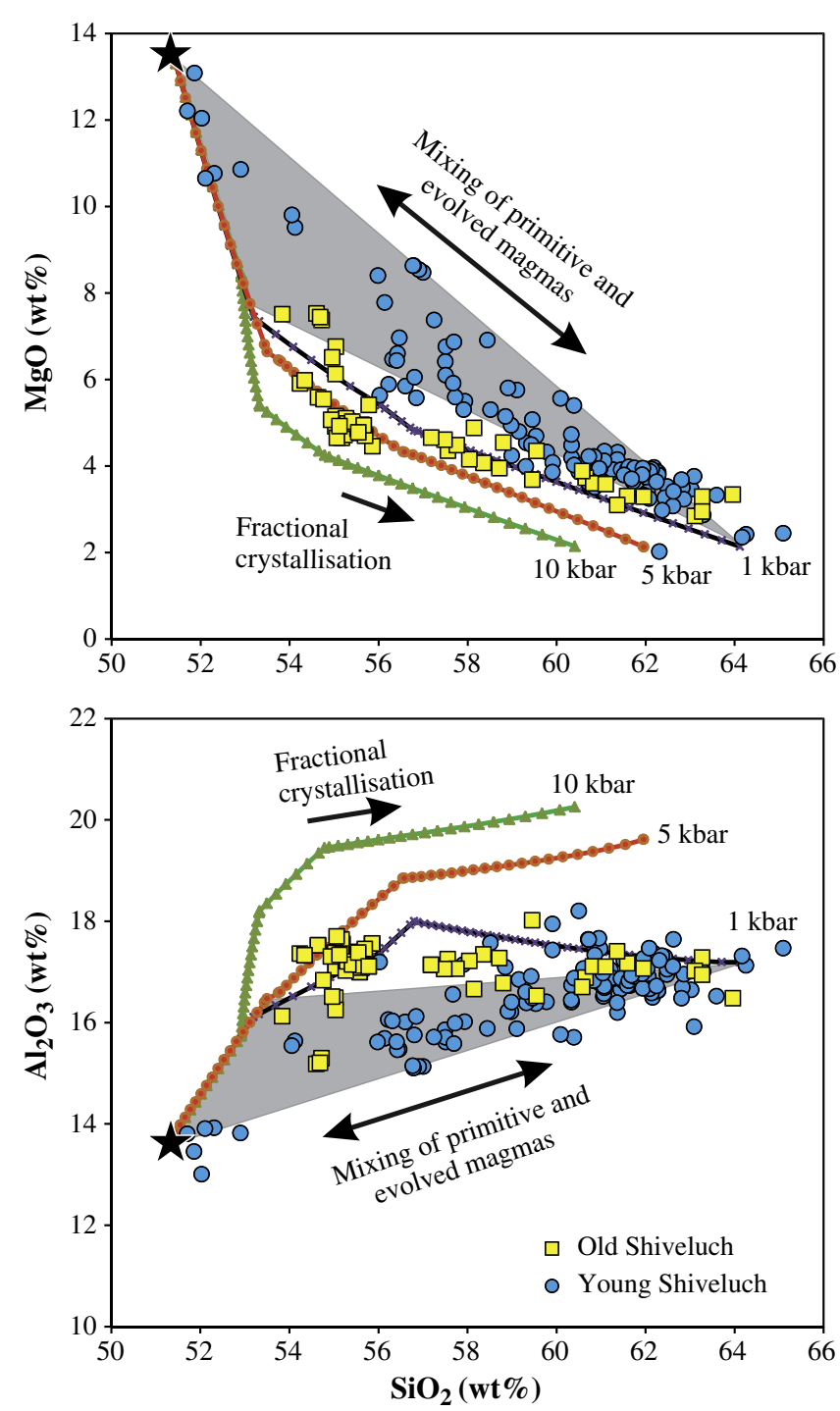

Fig. 12. Results of numeric simulation of fractional crystallization of primary Shiveluch magma at pressures of $0.1,0.5$ and $1.0 \mathrm{GPa}$. Input parameters are explained in Section 6.4. Data sources are listed in Fig. 9 caption. The composition of the majority of Old Shiveluch basaltic andesites and andesites as well as the composition of the most $\mathrm{SiO}_{2}$-rich Young Shiveluch andesites can be explained by fractional crystallization of basaltic primary melt at middle to shallow crustal conditions (pressure less than $\sim 0.5 \mathrm{GPa}$ ). The composition of high-Mg\# basaltic andesites cannot be explained by fractional crystallization of parental basaltic magma. It is suggested in this work and by Gorbach and Portnyagin (2011), who describe andesites of Young Shiveluch in great details, that the linear trends of Young Shiveluch rocks are best explained by mixing between primitive basaltic magmas with $\mathrm{MgO}=8-12 \mathrm{wt}$.\% and evolved andesites with $\mathrm{MgO}<2 \mathrm{wt} . \%$. Primary high-Mg\# andesitic melt is not required to explain the petrographic and geochemical features of Young Shiveluch rocks.

Shiveluch can represent products of $\sim 40 \%$ fractional crystallization of olivine, clinopyroxene and magnetite from primitive basaltic magma at $~ 0.5 \mathrm{GPa}$. Fractionation of plagioclase is not required to explain the origin of these rocks, and their abundant plagioclase phenocrysts could crystallize from the magmas at lower pressures but not removed from the magma until eruption. More evolved Old Shiveluch magmas overlapping with the compositions of Young Shiveluch andesites deviate to low pressure cotectics and can represent products of extensive ( $>60 \%$ ) fractional crystallization of primary basalt at pressures of $\sim 0.1 \mathrm{GPa}$ corresponding to shallow crustal depths $(\sim 3 \mathrm{~km})$ in a good agreement with previous estimates for Young Shiveluch andesites (e.g., Dirksen et al., 2006; Humphreys et al., 2006; Gorbach and Portnyagin, 2011). 
High-Mg basaltic andesites of Young Shiveluch cannot be obtained by fractional crystallization of the basaltic parent at any pressure and deviate from the cotectic compositions to higher $\mathrm{SiO}_{2}$ and lower $\mathrm{Al}_{2} \mathrm{O}_{3}$ at given $\mathrm{MgO}$ content. Because there is no direct evidence for the existence of primary andesitic magmas beneath Shiveluch (Fig. 8c), the linear trends of major elements can be well explained by mixing of primitive basalts with $\mathrm{MgO}=8-12 \mathrm{wt} . \%$ and $\mathrm{SiO}_{2}=50-53 \mathrm{wt} . \%$ and evolved andesites or andesites with $\mathrm{MgO}<2$ wt.\% and $\mathrm{SiO}_{2}>62$ wt.\%. (Fig. 12). Unequivocal field, petrographic and geochemical evidence for hybridism of primitive and evolved magmas in the plumbing system of Young Shiveluch has been shown by Gorbach and Portnyagin (2011). The evidence of the magma mixing was also documented in lava of 2001-2004 eruption (Humphreys et al., 2006; Dirksen et al., 2006; Gorbach, 2006), in some pumice of 1964 eruptions (Ivanov, 2008) and in more ancient pumices of Young Shiveluch (Volynets, 1979). Magma mixing could also contribute to the compositional diversity of the Old Shiveluch rocks, in particular to create the scatter of $\mathrm{MgO}, \mathrm{Al}_{2} \mathrm{O}_{3}, \mathrm{TiO}_{2}$, $\mathrm{K}_{2} \mathrm{O}, \mathrm{Cr}$, and $\mathrm{Ni}$ at a given $\mathrm{SiO}_{2}$ in the group of high-Mg basaltic andesites erupted at the end of the initial stage. Magma hybridism was however a subordinate process compared to fractional crystallization to generate the magma series of Old Shiveluch.

A major change in the composition of Shiveluch magmas was thus increasing amount of magnesian basaltic andesites and andesites on the Holocene stage of the volcano evolution. Whereas the majority of Old Shiveluch magma compositions can be explained by fractional crystallization of basaltic parental magma at middle to shallow crustal depths, magnesian basaltic andesites and andesites of Young Shiveluch originate predominantly by mixing of primitive basaltic and evolved andesitic to dacitic magmas.

\subsection{The potential effect of sector collapse on Shiveluch magmatic evolution}

Many volcanoes worldwide show a clear response of volcanic activity and magma plumbing system to sector collapse events. Eruptions of Bezymianny in 1956 and Mount St. Helens in 1980 are probably the best examples that a sharp release of lithostatic pressure due to edifice collapse can provoke a violent explosive eruption. A large scale collapse with a volume of tens $\mathrm{km}^{3}$ force magmatic plumbing system to adjust to new conditions and can lead to significant changes in the magma transport dynamics and the ways of magma differentiation (e.g., Pinel and Jaupart, 2000, 2003, 2005). Eruptive history and geochemical evolution of many volcanoes recorded an apparent change of lava composition after large scale sector collapses which caused changes of magma recharge rates, degree of fractionation and intensified magma mixing processes (Amelung and Day, 2002; Manconi et al., 2009). These effects are most clearly expressed on ocean-island volcanoes (e.g., Manconi et al., 2009; Longpre et al., 2009 and references therein), where the decompression following surface unloading due to giant collapses could extend even to the mantle level (Presley et al., 1997). Several arc stratovolcanoes (e.g., Stromboli, Italy; Parinacota, Chili) also exhibited change in dynamics of volcanic activity that involved changes in magma chamber processes (Tibaldi, 2004; Ginibre and Wörner, 2007).

Our data show that the composition of erupted magmas changed significantly after the large scale collapse which destroyed the Old Shiveluch edifice in the Late Pleistocene. The study of melt inclusions in Shiveluch tephra erupted from $\sim 16$ to $11 \mathrm{ka}$ BP also shows significant variations of the melt composition, allowing to make an assumption about the adjustment of Shiveluch magma plumbing system (Pevzner et al., 2012). The causal link between the sector collapse and the change of magma compositions and relative timing of these processes remain, however, uncertain because the age, scale and mechanism of the sector collapse are still subject to debate. The volume estimates of the destructed and displaced material due to the Shiveluch sector collapse range from 30 to $100 \mathrm{~km}^{3}$ (Melekestsev et al., 1991; Ponomareva et al., 2006; Pevzner et al., 2012). The data about the age of this event also are controversial. According to earlier work, the collapse occurred before the Last Glacial Maximum (2324 ka BP, Melekestsev et al., 1991), and in this case the decompression after surface unloading could be compensated by the following ice load. Other authors suggested that the collapse occurred after the last glaciation (10 ka, Belousov et al., 1999) or during the glaciation (15-16 ka, Pevzner et al., 2012). Thus, in order to discuss the influence of the collapse and deglaciation events on the Shiveluch magmatic evolution, more precise data are required on how these processes were correlated in time.

Nevertheless, despite the lack of consensus on the scale and the age of the Old Shiveluch sector collapse, this event is the largest known in Kamchatka (Melekestsev et al., 1991; Ponomareva et al., 2006) even if one assumes the minimum volume estimate. The increased role of magma mixing in the origin of the Young Shiveluch magmas may reflect important changes in the magma plumbing system beneath Shiveluch following the sector collapse. It cannot be excluded that a deep-rooted Old Shiveluch magma plumbing system has been adjusted and then diminished and/or destroyed after the sector collapse. The Holocene magma feeding system appears to have restricted to a smaller and more evolved magma chamber allowing direct mixing of evolved andesitic and primitive basaltic magmas from depth on the Holocene stage of the volcano evolution.

\section{Conclusions}

Late Pleistocene Old Shiveluch volcano was constructed during two major stages: initial pyroclastic stage and subsequent effusive stage. The initial agglomerate and psephytic pyroclastic deposits form at least $60 \%$ of the Old Shiveluch volcanic edifice. Similar lithology and spatial distribution of the initial Old Shiveluch and Young Shiveluch pyroclastic deposits suggest their similar eruptive styles although the Late Pleistocene eruptions likely had much larger scale.

The Old Shiveluch lava complex was formed by lavas from four major vents. The position of the vents was reconstructed along the rim of the collapse crater. The most voluminous andesitic eruptions were associated with the Central and Western vents, which are located in the central part of the volcanic edifice and erupted simultaneously. The Baidarny and Southern vents have younger age, were located to the south from the summit area and erupted basaltic andesite lavas. The eruptive vents and dike swarms are spatially restricted to the destroyed southern sector of the volcanic edifice that suggests that the direction of the Old Shiveluch sector collapse was determined by the pre-existing stress field.

The change of the eruptive style from the initial extrusive-explosive to effusive eruptions was not accompanied by a significant change in the rock composition. Both pyroclastic and effusive Old Shiveluch stages are characterized by the predominance of magnesian andesites. During the initial phase, the magnesian andesites were associated with a small volume of high-Mg basaltic andesites. During the effusive stage, magnesian andesites erupted coevally with high-Al basaltic andesites. The volume ratio of all andesites and basaltic andesites in the Old Shiveluch edifice is estimated to be $4: 1$.

The Old Shiveluch rocks are compositionally distinct from the present-day Young Shiveluch andesites by having lower Mg\#, MgO, $\mathrm{Cr}$ and $\mathrm{Ni}$, and higher $\mathrm{FeO}^{\mathrm{T}}$ and $\mathrm{V}$ contents at given $\mathrm{SiO}_{2}$ and can originate by fractional crystallization of primary basaltic magma with subordinate role of mixing of primitive and evolved magmas. The latter process becomes to be a major petrogenetic process for Young Shiveluch volcano. The changes in the magma differentiation process since the Late Pleistocene could be related to the reorganization of the multi-level magma plumbing system beneath Old Shiveluch, which adjusted to a major sector collapse and evolved to a more simple shallow plumbing system beneath Young Shiveluch. 


\section{Acknowledgments}

We are very grateful to Raya Sagitova, Sergey Samoilenko, Tanya Manevich and Alexander Sokorenko for an invaluable help during the field work. Mario Thöner and Dagmar Rau are thanked for their help with analytical studies in GEOMAR. Gene Yogodzinski is dearly thanked for analyzing subset of samples by XRF. We are thankful to Vera Ponomareva, Ivan Melekestsev (Institute of Volcanology and Seismology, Far East Branch, Russian Academy of Sciences) and Maria Pevzner (Geological Institute, Russian Academy of Sciences) for their inspiring comments and valuable discussions about the Shiveluch geology. The authors are grateful to Carmelo Ferlito and an anonymous reviewer for their constructive comments which helped us to improve the quality of this manuscript.

This work was supported by grant \#s 07-III-D-08-094, 07-III-B-08-095 and 09-III-A-08-422 of the Far-East Branch of the Russian Academy of Sciences, grant \# 11-05-98555p_vostok_a from the Russian Foundation for Basic Research and Far-East Branch, Russian Academy of Sciences and a Russian-German joint project KALMAR supported by the Ministries of Science and Education of Germany (BMBF) and Russian Federation.

\section{References}

Acocella, V., Neri, M., 2009. Dike propagation in volcanic edifices: overview and possible developments. Tectonophysics 471, 67-77.

Almeev, R., Kimura, J.-I., Ariskin, A., Ozerov, A., 2013. Decoding crystal fractionation in calc-alkaline magmas from the Bezymianny Volcano (Kamchatka, Russia) using mineral and bulk rock compositions. Journal of Volcanology and Geothermal Research 263, 140-170

Amelung, F., Day, S., 2002. InSAR observations of the 1995 Fogo, Cape Verde, eruption: implications for the effects of collapse events upon island volcanoes. Geophysical Research Letters 29 (12), 1606.

Ariskin, A.A., Barmina, G.S., 1999. An empirical model for the calculation of spinel-mel equilibria in mafic igneous systems at atmospheric pressure: 2.Fe-Ti oxides. Contributions to Mineralogy and Petrology 134, 251-263.

Belousov, A., Belousova, M., Voight, B., 1999. Multiple edifice failures, debris avalanches and associated eruptions in the Holocene history of Shiveluch volcano, Kamchatka, Russia. Bulletin of Volcanology 61, 324-342.

Churikova, T., Dorendorf, F., Woerner, G., 2001. Sources and fluids in the mantle wedge below Kamchatka, evidence from across-arc geochemical variation. Journal of Petrology 42 (8), 1567-1594.

Danyushevsky, L.V., 2001. The effect of small amounts of $\mathrm{H}_{2} \mathrm{O}$ on crystallisation of midocean ridge and back-arc basin basalts. Journal of Volcanology and Geotherma Research 110, 265-280.

Danyushevsky, L.V., Plechov, P., 2011. Petrolog3: integrated software for modeling crystallization processes. Geochemistry, Geophysics, Geosystems 12, Q07021 (doi:07010.01029/02011GC003516).

Dirksen, O., Humphreys, M.C.S., Pletchov, P., Melnic, O., Demyanchuk, Y., Sparks, R.S.J., Mahony, S., 2006. The 2001-2004 dome-forming eruption of Shiveluch Volcano, Kamchatka: observation, petrological investigation and numerical modeling. Journal of Volcanology and Geothermal Research 155, 201-226.

Ermakov, V., 1985. Geology and eruptive history of Shiveluch volcano. Volcanism and Associated Processes. Abstract Volume of VI Volcanological Meeting, PetropavlovskKamchatsky, pp. 42-44 (In Russian).

Ferlito, C. 2011. Bimodal geochemical evolution at Sheveluch stratovolcano, Kamchatka, Russia: consequence of a complex subduction at the junction of the Kuril Kamchatka and Aleutian island arcs. Earth-Science Reviews 105 (1-2), 49-69.

Gill, J.B., 1981. Orogenic Andesites and Plate Tectonics. Springer-Verlag, Berlin-Heidelberg (390 pp.).

Ginibre, C., Wörner, G., 2007. Variable parent magmas and recharge regimes of the Parinacota magma system (N. Chile) revealed by $\mathrm{Fe}, \mathrm{Mg}$ and $\mathrm{Sr}$ zoning in plagioclase. Lithos 98, 118-140.

Gorbach, N.V., 2006. New lava of Shiveluch volcano: en evidense of magma mingling? 5rd Biennial Workshop on Subduction Processes Emphasizing the Japan-KurileKamchatka-Aleutian Arcs (JKASP-5), Japan, pp. 133-137.

Gorbach, N.V., Portnyagin, M.V., 2011. Geology and petrology of the lava complex of Young Shiveluch volcano, Kamchatka. Petrology 19 (2), 140-172 (Engl. Transl.).

Humphreys, M.C.S., Blundy, J.D., Sparks, R.S.J., 2006. Magma evolution and opensystem processes at Shiveluch Volcano: insights from phenocryst zoning. Journal of Petrology 47 (12), 2303-2334.

Ivanov, B.V., 2008. Andesites of Kamchatka. Reference guide to the chemical analyses of volcanites and basic rock-forming minerals. Nauka Press, Moscow (364 pp.)

Kress, V.C., Carmichael, I.S.E., 1988. Stoichiometry of the iron oxidation reaction in silicate melts. American Mineralogist 73, 1267-1274.

Levin, V., Park, J., Brandon, M., Lees, J., Peyton, V., Gordeev, E., Ozerov, A., 2002. Crust and upper mantle of Kamchatka from teleseismic receiver functions. Tectonophysics 358 (1-4), 233-265.
Longpre, M.-A., Troll, V.R., Walter, T.R., Hansteen, T.H., 2009. Volcanic and geochemical evolution of the Teno massif, Tenerife, Canary Islands: some repercussions of giant landslides on ocean island magmatism. Geochemistry, Geophysics, Geosystems 10 (12) (31 pp.).

Lopatin, V. B., Litvinov, A. F., Tsikunov, A.G., 1979. Gelology and mineral resources of the 0-57-XXV, XXXVI sheets (report on geological mapping and procpecting in 1:200000 scale). V. 1.252 pp. (in Russian).

Manconi, A., Longpré, M.-A., Walter, T.R., Troll, V.R., Hansteen, T.H., 2009. The effects of flank collapses on volcano plumbing systems. Geology 37, 1099-1102.

Melekestsev, I.V., Volynets, O.N., Ermakov, V.A., Kirsanova, T.P., Masurenkov Yu., P. 1991. Shiveluch volcano. In: Fedotov, S.A., Masurenkov Yu., P. (Eds.), Active Volcanoes of Kamchatka, 1. Nauka Press, Moscow, pp. 84-92.

Menyailov, A.A., 1955. Shiveluch Volcano, its geologic structure, composition and eruptions. Trudi Laboratorii Vulkanologii 9264 pp. (in Russian).

Münker, C., Wörner, G., Yogodzinski, G.M., Churikova, T., 2001. Behaviour of high field strength elements in subduction zones: constraints from Kamchatka-Aleutian arc lavas. Earth and Planetary Science Letters 224, 275-293.

Nakamura, K., 1977. Volcanoes as possible indicators of tectonic stress orientation: principle and proposal. Journal of Volcanology and Geothermal Research 2, 1-6.

Nikulin, A., Levin, V., Carr, M., Herzberg, C., West, M., 2012. Evidence for two upper mantle sources driving volcanism in Central Kamchatka. Earth and Planetary Science Letters 321-322, 14-19. http://dx.doi.org/10.1016/j.epsl.2011.1012.1039.

Pevzner, M.M., Babanskii, A.D., Tolstykh, M.L., Kononkova, N.N., 2012. The restructing of magma plumbing system Shiveluch volcanic massif as a consequence the large scale sector collapses in Late Pleistocene-Early Holocene. Doclady Academii Nayk 447 (3), 318-321 (In Russian).

Pinel, V., Jaupart, C., 2000. The effect of edifice load on magma ascent beneath a volcano. In: Francis, P., Neuberg, J., Sparks, R.S. (Eds.), The Causes and Consequences of Eruptions of Andesite Volcanoes; Papers of a Discussion Meeting: Philosophical Transactions - Royal Society. Mathematical, Physical and Engineering Sciences, 358, pp. 1515-1532.

Pinel, V., Jaupart, C., 2003. Magma chamber behavior beneath a volcanic edifice. Journal of Geophysical Research 108, 2072-2088.

Pinel, V., Jaupart, C., 2005. Some consequences of volcanic edifice destruction. Journal of Volcanology and Geothermal Research 145, 68-80.

Ponomareva, V.V., Melekestsev, I.V., Dirksen, O.V., 2006. Sector collapses and large landslides on Late Pleistocene-Holocene volcanoes in Kamchatka, Russia. Journa of Volcanology and Geothermal Research 158, 117-138.

Ponomareva, V.V., Kyle, P., Pevzner, M.M., Sulerzhitsky, L.D., Hartman, M., 2007. Holocene eruptive history of Shiveluch Volcano, Kamchatka Peninsula, Russia. In: Eichelberger, J., Gordeev, E., Izbekov, P., Lees, J. (Eds.), Volcanism and Subduction: The Kamchatka region: AGU Geophysical Monograph, 172, pp. 263-282.

Portnyagin, M., Manea, V.C., 2008. Mantle temperature control on composition of arc magmas along the Central Kamchatka Depression. Geology 36 (7), 519-522.

Portnyagin, M., Hoernle, K., Avdeiko, G., Hauff, F., Werner, R., Bindeman, I., Uspensky, V., Garbe-Schonberg, D., 2005. Transition from arc to oceanic magmatism at the Kamchatka-Aleutian junction. Geology 33 (1), 25-28.

Portnyagin, M.V., Bindeman, I.N., Hoernle, K., Hauff, F., 2007. Geochemistry of primitive lavas of the Central Kamchatka Depression: magma genesis at the edge of the Pasific Plate. In: Eichelberger, J., Gordeev, E., Izbekov, P., Lees, J. (Eds.), Volcanism and Subduction: The Kamchatka Region: AGU Geophysical Monograph, 172, pp. 199-239.

Portnyagin, M.V., Sobolev, A.V., Mironov, N.L., Hoernle, K., 2009. Pyroxenite melts involved in magma genesis in Kamchatka. 19th Annual V.M. Goldschmidt Conference, Davos, Switzerland, June 21-26: Geochimica Cosmochimica Acta, 73 (13, Supp. 1), p. A1044

Presley, T.K., Sinton, J.M., Pringle, M., 1997. Postshield volcanism and catastrophic mass-wasting of the Waianae volcano, Oahu, Hawaii. Bulletin of Volcanology 58 , 597-616.

Simakin, A.G., Chassemi, A., 2009. The role of magma chamber-fault interaction in caldera forming eruptions. Bulletin of Volcanology 72 (1), 85-101.

Tibaldi, A., 2004. Magor change in volcano behaviour after a sector collapse: insights from Stromdoly, Italy. Terra Nova 16, 2-8.

Volynets, O.N., 1979. Heterotaxitic lavas and pumice. Problems of Deep Magmatism. Nauka Press, Moscow, pp. 181-196 (In Russian).

Volynets, O.N., 1994. Geochemical types, petrology, and genesis of Late Cenozoic volcanic rocks from the Kurile-Kamchatka island-arc system. International Geology Review 36, 373-405.

Volynets, O.N., Ponomareva, V.V., Babanskii, A.D., 1997. Magnesian basalts of Shiveluch andesite volcano, Kamchatka. Petrology 5 (2), 183-196 (Engl. Transl.)

Volynets, O.N., Melekestsev, I.V., Ponomareva, V.V., Yogodzinksi, G.M., 1999 Kharchinskii and Zarechnyi volcanoes, unique centers of Late Pleistocene magnesian basalts in Kamchatka: rock composition. Volcanology and Seismology 1 31-45 (in Russian)

Yogodzinksi, G.M., Lees, J.M., Churikova, T.G., Dorendorf, F., Woerner, G., Volynets, O.N., 2001. Geochemical evidence for the melting of subducting oceanic lithosphere at plate edges. Nature 409, 500-504.

Yogodzinski, G.M., Volynets, O.N., Koloskov, A.V., Seliverstov, N.I., Matvenkov, V.V. 1994. Magnesian andesites and the subduction component in a strongly calcalkaline series at Piip volcano, Far Western Aleutians. Journal of Petrology 35 (1), 163-204.

Yogodzinski, G.M., Kay, R.W., Volynets, O.N., Koloskov, A.V., Kay, S.M., 1995. Magnesian andesite in the western Aleutian Komandorsky region: implications for slab melting and processes in the mantle wedge. Geological Society of America Bulletin 107 (5), 505-519. 